Staff Working Paper/Document de travail du personnel 2016-33

\title{
Relationships in the Interbank Market
}

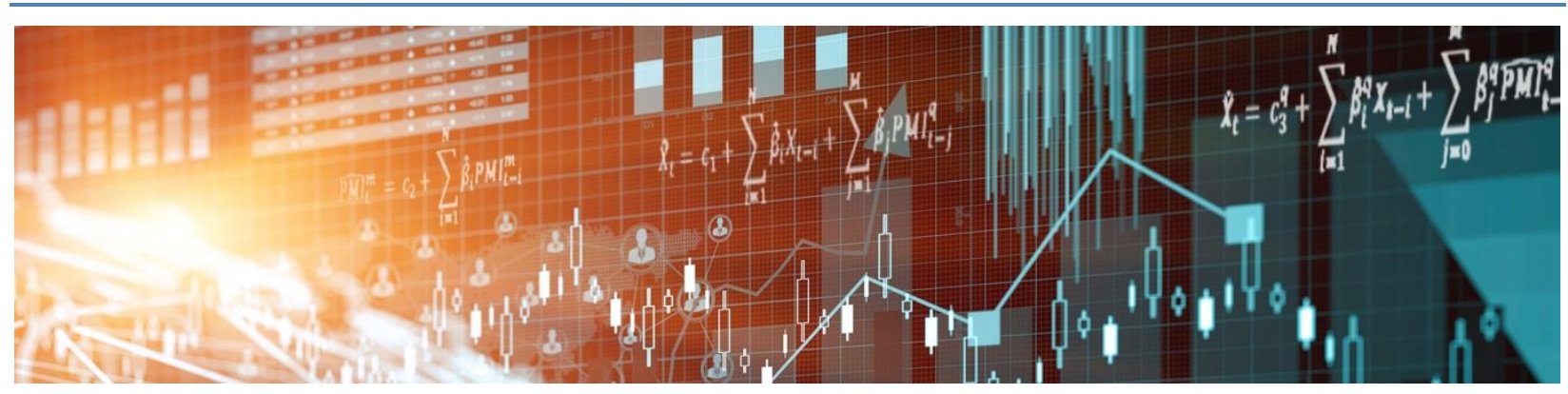

by Jonathan Chiu and Cyril Monnet 
Bank of Canada Staff Working Paper 2016-33

July 2016

\title{
Relationships in the Interbank Market
}

\author{
by \\ Jonathan Chiu ${ }^{1}$ and Cyril Monnet ${ }^{2}$ \\ 1 Victoria University of Wellington and \\ Funds Management and Banking Department \\ Bank of Canada \\ Ottawa, Ontario, Canada K1A 0G9 \\ Jonathan.Chiu@vuw.ac.nz \\ 2University of Bern, Study Center Gerzensee \\ Swiss National Bank \\ cyril.monnet@vwi.unibe.ch
}




\section{Acknowledgements}

We thank Morten Bech and Marie Hoerova for many useful conversations. We thank Luis Araujo, Todd Keister, Thomas Nellen, Peter Norman, Guillaume Rocheteau, Alberto Trejos, Pierre-Olivier Weill, Randall Wright, Shengxing Zhang, and audiences at Queen's University; the University of Helsinki; the Vienna Macro Workshop; the Summer Money, Banking and Liquidity Workshop at the Chicago Fed; the Norges Bank; the Riksbank; and the second African Search and Matching Workshop for useful comments.

The views expressed here are those of the authors and do not necessarily reflect the position of the Bank of Canada or the Swiss National Bank. 


\begin{abstract}
The market for central bank reserves is mainly over-the-counter and exhibits a core-periphery network structure. This paper develops a model of relationship lending in the unsecured interbank market. In equilibrium, a tiered lending network arises endogenously as banks choose to build relationships to insure against liquidity shocks and to economize on the cost to trade in the interbank market. Relationships matter for banks' bidding strategies at the central bank auction and introduce a relationship premium that can significantly distort the observed overnight rate. For example, it can explain some anomalies in the level of interest rates-namely, that banks sometimes trade above (below) the central bank's lending (deposit) rate. The model also helps to explain how monetary policy affects the network structure of the interbank market and its functioning, and how the market responds dynamically to an exit from the floor system. We also use the model to discuss the potential effects of bilateral exposure limits on relationship lending.
\end{abstract}

JEL Codes: E4, E5

Bank topics: Interest rates; Monetary policy implementation; Transmission of monetary policy

\title{
Résumé
}

Le marché des réserves de banques centrales est, dans la plupart des cas, un marché de gré à gré dont la structure de réseau est de type « centre-périphérie ». Les auteurs modélisent les relations contractuelles de long terme dans le marché des prêts interbancaires non garantis. Les banques choisissent d'établir des relations pour se protéger contre des chocs de liquidité et économiser les coûts des opérations de marché. En situation d'équilibre, un réseau de prêt par paliers est donc généré de façon endogène. Les relations interbancaires sont importantes pour comprendre les stratégies de soumission des banques dans le cadre des processus d'adjudication des réserves des banques centrales. Par ailleurs, les banques en relation incorporent une prime de relation dans les taux qu'elles négocient, ce qui peut fausser le taux de refinancement à un jour. Ces primes peuvent, par exemple, expliquer certaines anomalies du niveau des taux d'intérêt - à savoir, le fait que les banques pratiquent parfois des taux supérieurs aux taux d'escompte des banques centrales ou inférieurs aux taux de rémunération des dépôts de ces dernières. Le modèle aide également à expliquer l'influence qu'exerce la politique monétaire sur la structure de réseau et le fonctionnement du marché des prêts interbancaires ainsi que la réaction dynamique des marchés lorsque les banques centrales mettront fin à leurs politiques monétaires non conventionnelles. Les auteurs utilisent aussi le modèle pour analyser les effets des nouvelles réglementations limitant l'exposition au risque de crédit sur les relations bancaires de long terme.

\section{Classification JEL : E4, E5}

Classification de la Banque : Taux d'intérêt, Mise en œuvre de la politique monétaire, Transmission de la politique monétaire 


\section{Non-Technical Summary}

Major central banks now implement monetary policy by using a corridor/channel system to influence interest rates in the interbank market. Contrary to popular belief, the interbank market is far from being the epitome of a perfectly competitive market. Instead, it is mainly over-thecounter and exhibits a core-periphery network structure.

This paper develops a model of relationship lending in the unsecured interbank market. In equilibrium, a tiered lending network arises endogenously as banks choose to build relationships to insure against liquidity shocks and to economize on the cost to trade in the interbank market. Relationships matter for banks' bidding strategies at the central bank auction. They also introduce a relationship premium that can significantly distort the observed overnight rate. For example, relationships can explain some anomalies in the level of interest rates-namely, that banks sometimes trade above (or below) the central bank's lending (deposit) rate.

A lesson for policy-makers is that trades outside the corridor are consistent with a wellfunctioning interbank market, even when all banks have access to the facilities. Thus, the central bank has no need to worry about eliminating deviations due to long-term relationships. We should be careful, however, in interpreting the interbank market rate as a reference for the overnight cost of liquidity because it may also incorporate a relationship premium, which at times can significantly distort the observed overnight rate.

The model also helps with our understanding of how monetary policy affects the network structure of the interbank market and its functioning, as well as how the market responds dynamically to an exit from the floor system. Another way we use the model is to discuss the potential effects of bilateral exposure limits on relationship lending. 


\section{Introduction}

Major central banks implement monetary policy by targeting the overnight rate in the unsecured segment of the interbank market for reserves - the very short-term rate on the yield curve. The textbook principles of monetary policy implementation are intuitive: Each bank holds a reserve account at the central bank. Over the course of a normal business day, this account is subject to shocks due to the banks' payment inflows or outflows. Banks seek to manage their account balance to satisfy some forms of reserve requirements. ${ }^{1}$ The central bank manages the supply of reserves by conducting auctions or open market operations. By changing the supply of reserves, it influences the interest rate at which banks borrow or lend reserves in the interbank market, and, as a consequence, the marginal cost of making loans to businesses and individuals. $^{2}$ In recent years, many major central banks have refined this system by offering two facilities: In addition to auctioning reserves, the central bank stands ready to lend reserves at a penalty rate - the lending rate - if banks end up short of reserves. Symmetrically, banks can earn an interest rate - the deposit rate - if they end up holding reserves in excess of the requirement. As a consequence the interbank rate should stay within the bands of the corridor defined by the lending and the deposit rates. This is known as the "corridor system" for monetary policy implementation.

The reality is more complex than this basic narrative. On many occasions, central banks overestimated the demand for liquidity needed for banks to satisfy their reserve requirements (see for example European Central Bank, 2002). Bowman, Gagnon, and Leahy (2010) and others report that in many jurisdictions with large excess reserves, banks have been trading below the deposit rate (supposedly the floor of

\footnotetext{
${ }^{1}$ The minimum reserve requirement can be either positive (e.g., in the United States) or zero (e.g., in Canada).

${ }^{2}$ For example, in normal circumstances, the European Central Bank conducts weekly auctions, while the Fed conducts repos with its primary dealers. Tha Bank of Canada conducts open market opeations and also auctions settlement balances.
} 
the corridor). ${ }^{3}$ It is also well known that banks sometimes trade above the lending rate (supposedly the ceiling of the corridor). This challenges the basic intuition that simple arbitrage would maintain the rates within the bands of the corridor. At a time when central banks are thinking of exiting quantitative easing policies, we may wonder whether these observations are symptomatic of a dysfunctional interbank market that will hamper exit, or if they are "natural" phenomena with little relevance for the conduct of monetary policy during the exit stage.

Contrary to folk belief, the interbank market is very far from being the epitome of the Walrasian market. For example, Figure 1 illustrates that a large share of the transactions in the European (unsecured) interbank market is made over-the-counter (OTC). Also, many banks maintain long-term relationships and they trade with just a few banks, sometimes only one, or they directly access the central bank facilities without even trading with another bank. We review the evidence below, but these facts are now well accepted.

In this paper, we analyze the effects of long-term trading relationships and mon-

\footnotetext{
${ }^{3}$ Bowman, Gagnon and Leahy (2010) review the experience of eight major central banks and report that the deposit rates on reserve do not always provide a lower bound for short-term market rates. In particular, the (weighted) average of overnight market rates for reserve balances sometimes stayed below the deposit rate during the recent financial crisis, when reserve balances were abundant and the central bank moved its overnight target towards the deposit rate on reserves. In some countries, a potential explanation for this puzzling observation is that some participants in the money market cannot earn interest on their deposits at the central bank (e.g., GSEs in the United States). In some other cases, there is no clear institutional feature that can explain why the average overnight rate stayed below the floor. For example, according to the above study, in Japan, "[t]he uncollateralized overnight call rate is similar to the federal funds rate, in that it is a daily weighted average of transactions in the uncollateralized overnight market. Participants in the market include domestic city, regional, and trust banks, foreign banks, securities companies, and other firms dealing in Japanese money markets. All of the economically important participants active in the call market are also eligible to participate in the deposit facility...the overnight call rate has occasionally fallen below the Bank of Japan's deposit rate in the period since November 2008, but never by more than 2 basis points." Similarly, in Canada, there is anecdotal evidence that some banks occasionally lent and borrowed at rates below the floor during the crisis period, even though they had direct access to central bank deposit facilities. Concerning the explanation for these negative spreads in Canada, some practitioners argued that banks lent below the floor due to concerns about their reputation and relationship with other banks in the market (see Lascelles, 2009).
} 
Figure 1: Trading structure of unsecured transactions in the European interbank market (Source: ECB, 2013)

etary policy on bidding behavior, interbank trading volume and rates, the network structure of the interbank market and its functioning. We show that modeling relationships matters for both individual and aggregate demand for liquidity. Relationships can explain why banks trade below the deposit rate or above the lending rate. Our analysis also sheds light on the effect of monetary policy on the network structure. In particular, we show that the current accommodative monetary policy stance lowers the value of building and maintaining relationships, so that in steady state, no or few relationships exist. Interestingly, this is what some central bankers have been pointing out, and it arises endogenously from our model. ${ }^{4}$ Moreover, relation-

\footnotetext{
${ }^{4}$ Bech et al. (2014) find significant changes in lending patterns following the implementation of the interest on excess reserves policy by the Federal Reserve on October 9, 2008. Also, Klee and Stebunovsy (2013) report a large decline in fed funds volume in the second quarter of 2012, while Beltran, Bolotnyy, and Klee (2015) find that "many of the smaller lenders reduced their lending to the larger institutions in the core of the network (...) the federal funds network began to contract rapidly and change dramatically in structure after the Lehman bankruptcy in the fall of 2008 . The number of participating banks and transactions plummeted, as did overall dollar volume." Furthermore they show that "there was a sharp contraction in the number of links in the network during the last quarter of 2008 (...) Also, there was a sizable increase in the average dollar volume transacted per link, which (...) is largely driven by the exit of many of the smaller banks from the
} 
ships also matter for the effects of future policy changes. For example, we show how the interbank network responds dynamically to an exit from the floor system. We also discuss the potential effects on relationship lending of the regulation on bilateral exposure which will come into force in 2019.

We model the corridor system for monetary policy implementation under zero reserve requirements. ${ }^{5}$ At the beginning of each day, banks can bid for central bank reserves at the policy rate set by the central bank. This is akin to a tender with full allotment, like the one the European Central Bank has been operating since 2008. Once they obtain reserves at the tender, banks face a shock to their reserves holdings. To meet their reserve requirement, banks in a deficit position can borrow at the central bank's lending facility at $i_{\ell}$, and those in a surplus position can deposit their reserves at the deposit facility to earn $i_{d}$. A positive spread between $i_{\ell}$ and $i_{d}$ gives banks incentives to trade reserves directly with each other. These trades can be conducted either in the core interbank market where banks can trade with many different counterparties, or at the periphery of the core market by banks with preestablished relationships. Some banks (that we call $S$ for "small") have to pay a cost to access the core market, while others (that we call $L$ for "large") can access it freely. ${ }^{6}$ In addition, banks can trade reserves with their long-term partners in the relationship-lending market. Within a relationship, the $L$ bank can provide intermediation service to $S$ by accessing the core market on its behalf; the $S$ bank saves on the access cost, and the large bank extracts some rents from providing this service. Furthermore, a relationship allows long-term partners to trade repeatedly market."

${ }^{5}$ Setting the minimum reserve requirement at zero is just a normalization. Our finding does not rely on this assumption.

${ }^{6}$ For example, small banks usually do not have a liquidity manager and so their opportunity cost of accessing the core market is high. Hence, small banks will only enter the core market if their gains from trade are sufficiently large. Also, "small" and "large" are merely labels for banks with high and low participation costs. We will show that, in equilibrium, low-cost banks will trade larger volume than high-cost banks in the interbank market, hence justifying the labels "large" and "small." 
over time without the need to search for a new counterparty everyday. However, relationships can end for exogenous or endogenous reasons. In that case, banks can search for another long-term partner to build a new relationship.

Within this framework, the corridor can be "soft," i.e., equilibrium interbank rates can be below $i_{d}$ or above $i_{\ell}$, when trading frictions are present, even though small banks can access the deposit/lending facilities at no cost. In other words, it seems that arbitrage opportunities exist. The intuition is clear: small banks value long-term relationships, as they provide liquidity insurance and save them the cost of accessing the core market. As a result, they are willing to temporarily lower their surplus from trading a loan as long as the long-term gains from keeping a relationship outweigh the short-term loss. This gives rise to what we call a relationship premium. ${ }^{7}$ Specifically we show that $S$ banks with surplus reserves agree to lend at a rate below $i_{d}$, when this helps them keep their relationship. Symmetrically, $S$ banks that need reserves may end up paying a rate above $i_{\ell}$. Therefore, in equilibrium, some banks trade below the floor or above the ceiling of the corridor. On the surface, there seems to be unexploited arbitrage opportunities. There is none really: $S$ banks are only willing to trade at a rate outside the corridor for small loans with their long-term partners, but not for large loans or with other counterparties.

Furthermore, our model implies that the soft floor depends on aggregate liquidity conditions: It is more likely when there is a large aggregate liquidity surplus or deficit. A lesson for policy-makers is that trades outside the corridor are consistent with a well-functioning interbank market, even when all banks have access to the facilities. Thus, the central bank has no need to worry too much about eliminating deviations due to long-term relationships. However, one should be careful in interpreting the interbank market rate as a reference for the overnight cost of liquidity, because it

\footnotetext{
${ }^{7}$ This implication is also consistent with the finding by Ashcraft and Duffie (2007) that "[t]he rate negotiated is higher for lenders who are more active in the federal funds market relative to the borrower. Likewise, if the borrower is more active in the market than the lender, the rate negotiated is lower, other things equal."
} 
may also incorporate a relationship premium, which at times can significantly distort the observed overnight rate.

Our work is also one of the first to attempt to explain the network structure of the interbank market and its endogenous response to a change in monetary policy. The network structure that emerges endogenously resembles the core-periphery structure we observe in the data, where most of the trading activities are due to a number of banks that appear to intermediate the trades of others. We show that as monetary policy becomes more accommodative, banks have little gains from their relationships: Since reserves are cheap, they have enough reserves to satisfy their requirements under most circumstances and the gains from building relationships and conducting relationship trades become small. The tiered network structure will then slowly vanish, as banks are hit by exogenous separation shocks. As the central bank gives up its accommodative stance, we show that the path to the new steady state is not necessarily monotonic: $S$ banks may have a large incentive to search for a relationship early on, and the high success rate explains why $S$ banks can reduce their search effort below its steady-state level.

\section{Literature}

Figure 2 shows the network of the federal funds market as shown by Bech and Atalay (2010) for September 29, 2006. The market has a core-periphery network structure (or tiered structure) where some banks in the periphery only trade with one bank, while the latter might trade with many others. ${ }^{8}$ Bech and Atalay (2010) point out that, in the US interbank market, "[t]here are two methods for buying and selling

\footnotetext{
${ }^{8}$ Sept. 292006 was the last day of the third quarter. Bech and Atalay report that a total of 479 banks were active in the market that day. The largest bank in terms of fed funds value traded is located at the center of the graph. The 165 banks that did business with the center bank lie on the first outer circle. The second outer circle consists of the 271 banks that did business with the banks in the first circle, but that did no business with the center bank. The remaining dots are the 42 banks that were more than two links away from the center. Two banks did business only between themselves. Large-value links are in yellow and small-value links are in red.
} 
federal funds. Depository institutions can either trade directly with each other or use the services of a broker. ... In the direct trading segment, transactions commonly consist of sales by small-to-medium sized banks to larger banks and often take place on a recurring basis. The rate is set in reference to the prevailing rate in the brokered market. In the brokered segment, participation is mostly confined to larger banks acting on their own or a customers behalf."

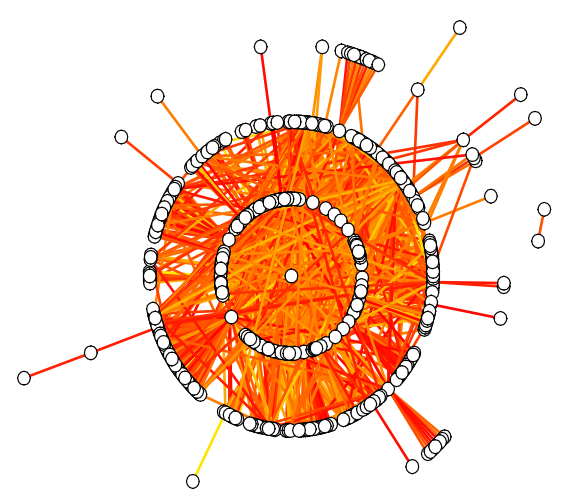

Figure 2: Network structure of the fed funds market (Bech and Atalay, 2010)

Stigum and Crescenzi (2007, Ch. 12) also report anecdotal evidence of the tiered structure of the fed funds market. In particular, they report that

"[i]n the fed funds market now, regional banks buy up funds from even tiny banks, use what they need, and resell the remainder in round lots in the New York market. Thus, the fed funds market resembles a river with tributaries: money is collected in many places and then flows through various channels into the New York market. In essence, the nation's smaller banks are the suppliers of fed funds, and the larger bankers are the buyers."

Also 
"[t]o cultivate correspondents that will sell funds to them, large banks stand ready to buy whatever sums these banks offer, whether they need all these funds or not. If they get more funds than they need, they sell off the surplus in the brokers market. Also, they will sell to their correspondents if the correspondents need funds, but that occurs infrequently. As a funding officer of a large bank noted, "We do feel the need to sell to our correspondents, but we would not have cultivated them unless we felt that they would be selling to us $99 \%$ of the time. On the occasional Wednesday when they need $\$ 100,000$ or $\$ 10$ million, OK. Then we would fill their need before we would fill our own."”

Elsewhere, using Bundesbank data on bilateral interbank exposures among 1,800 banks, Brauning and Fecht (2012) and Craig and Von Peter (2014) find strong evidence of tiering in the German banking system. Using UK data Vila et. al. (2010) also report the existence of a core of highly connected banks alongside a periphery. Of course, this has important consequences on rates. As Stigum and Crescenzi (2007) note,

"A few big banks, however, still see a potential arbitrage, 'trading profits,' in selling off funds purchased from smaller banks and attempt to profit from it to reduce their effective cost of funds. Also a few tend to bid low to their correspondents. Said a trader typical of the latter attitude, 'We have a good name in the market, so I often underbid the market by $1 / 16 . "$

Our paper is related to the literature on the interbank market and monetary policy implementation, to the growing literature on financial networks, and to the literature on OTC markets. The first literature on the interbank market includes Poole (1968), Hamilton (1996), Berentsen and Monnet (2006), Berentsen, Marchesiani, and Waller (2014), Bech and Klee (2011), Afonso and Lagos (2014), and Afonso, Kovner and 
Schoar (2012), among others. See also Bech and Keister (2012) for an interesting application of the Poole (1968) model to reserve management with a liquidity coverage ratio requirement. While Afonso, Kovner, and Schoar (2012) show some evidence of long-term relationship in the interbank market, none of the papers above accounts for it. Rather, they all treat banks as anonymous agents conducting random, "spot" trades. So our paper is the first to study the effect of long-term relationship on rates. Based on private information, Ennis and Weinberg (2013) explain why some banks can borrow at a rate above the central bank's lending rate. The early literature on financial networks is mostly motivated by understanding financial fragility and has been covered in Allen and Babus (2009); see also Jackson (2010). It includes Allen and Gale (2000) who study whether some banks' networks are more prone to contagion than others. Also, Leitner (2005) studies the optimality of linkages, motivated by the desirability of mutual insurance when banks can fail; while Gofman (2011) and Babus (2013) analyze the emergence and efficiency of intermediaries in OTC markets. In a recent calibration exercise, Gofman (2014) finds that it is suboptimal to limit banks' interdependencies in the interbank market. Elliott, Golub, and Jackson (2014) apply network theory to financial contagion through net worth shocks. Finally, the literature on OTC markets includes Duffie, Garleanu, and Pedersen (2005), and Lagos and Rocheteau (2006), among many others. ${ }^{9}$ Within this literature, Chang and Zhang (2015) study network formation in asset markets based on heterogeneous liquidity preferences.

There is a large empirical literature on the interbank market, and we already mentioned a few papers. Furfine (1999) proposes a methodology to extract fed funds transactions from payments data, and Armantier and Copeland (2012) test the methodology. Afonso, Kovner and Shoar (2011) study the fed funds market in times of stress. The two papers closest to ours are perhaps the empirical study of

\footnotetext{
${ }^{9}$ See also Afonso and Lagos (2014); Li, Rocheteau and Weill (2012); Lagos, Rocheteau, and Weill (2011); and Rocheteau and Wright (2013).
} 
Brauning and Fecht (2012) and the theoretical paper of Blasques, Brauning, and van Lelyveld (2015). Brauning and Fecht suggest a theory of relationship lending based on private information, as proposed by Rajan (1992). In good times, banks extract an informational rent, thus explaining why the relationship lending rates are usually higher than the average rate in normal times. In bad times, a lending bank knows whether the borrower is close to failure and it is willing to offer a discount in order to keep the bank afloat. This argument fails to recognize that in bad times, some borrowers may not be close to failure and the rent that can be extracted from a relationship lender can be even higher then. Moreover, the size of discount involved in these loans is usually not an amount significant enough to matter for the survival of a borrowing bank. Although we do not want to minimize the role of private information, we argue that the simple threat of terminating the relationship can also yield to interbank rate discounts. Blasques, Brauning, and van Lelyveld (2015) study a dynamic network model of the unsecured interbank market based on peer monitoring. However, they do not study the impact of the supply of reserves on the structure of the network, or explain why interest rates can fall outside of the corridor.

Section 2 describes the environment. For simplicity, we start with a basic model where the core interbank market is competitive and frictionless. Sections 3 and 4 characterize the equilibrium. In Section 5, we analyze the qualitative implications of this basic model. Section 6 then extends the model to incorporate an OTC interbank market and reports some quantitative implications of the extended model. We conclude in Section 7.

\section{Model}

We model the daily reserve management problem of banks in a corridor system. To study relationships and to determine their equilibrium values, we consider an infinite 
horizon model with discrete time. There is a central bank and an equal-measure (1/2) continuum of two types of banks, $S$ and $L$. A pair of $S$ and $L$ banks can form a relationship, which allows them to trade repeatedly until they are separated either exogenously or endogenously. ${ }^{10}$ All banks are infinitely-lived and discount the future at rate $\beta \in(0,1)$. They are subject to reserve requirements, and they have to hold at least $\bar{R}$ units of reserves at the end of each day. For simplicity we normalize $\bar{R}$ to zero. Each unit of reserve is a claim to a numeraire good. Banks are risk neutral and they enjoy utility $q$ from consuming $q$ units of the numeraire (where $q<0$ if they produce).

At the beginning of each period, banks settle their past interbank trades as well as their obligations towards the central bank by producing or consuming the numeraire good. Settlement is an automatic process and banks do not default, so all banks hold zero reserve balances once settlement takes place. ${ }^{11}$ In addition, some banks start the period in a relationship and are called matched banks. The rest are called single banks. At the beginning of each period, matched banks' current business relationship ends with probability $\sigma \in(0,1)$. Following settlement, there are four sub-periods $s=1,2,3,4$ in which banks can trade reserves. Figure 3 shows the timeline. ${ }^{12}$

In sub-period 1, the central bank operates a full allotment tender at rate $\bar{i}$. Hence, the discounted (gross) cost of borrowing $R$ units of reserves at the tender (in terms of the numeraire) is $\beta(1+\bar{i}) R$ since the loan is settled at the beginning of the next period. We allow $R<0$ to capture the feature of a liquidity-absorbing operation. ${ }^{13}$ Banks bid for reserves at the tender, and the central bank satisfies all individual bids fully. For simplicity, we assume the central bank grants unsecured loans.

\footnotetext{
${ }^{10}$ In our companion paper, we briefly discuss an extension with relationships between two $L$ banks and relationships between two $S$ banks.

${ }^{11}$ Our assumption on linear utility ensures that banks are indifferent between consuming the numeraire or carrying reserves forward.

${ }^{12}$ In the figure, black dots represent $L$ banks, red circles represent $S$ banks and arrows denote flows of funds.

${ }^{13}$ This is similar to what is referred to as a reverse auction.
} 
In sub-period 2, banks receive a liquidity shock. $S$ banks draw a shock $\xi$ from the distribution $F(\xi)$, while $L$ banks draw a shock $\varepsilon$ from the distribution $G(\varepsilon)$, which can potentially be different from $F$. Following this shock, a pair of banks already in a relationship can borrow from and lend to one another. These recurring bilateral links form the periphery of the core market, or the peripheral market. Banks use proportional bargaining, and $S$ banks obtain a share $\theta$ of the trading surplus. We call the interest rate $\hat{i}\left(R_{L}, R_{S}\right)$ of a loan between an $S$ bank holding $R_{S}$ reserves and an $L$ bank holding $R_{L}$ reserves the relationship rate. Single banks do not trade, ${ }^{14}$ but can build new relationships. Specifically, single $S$ banks can pay a cost $\kappa$ to call one $L$ bank to form a relationship starting from the next day (so the decision to call does not depend on the current shock). The call is random so that both single and matched $L$ banks can be approached. We assume that the probability that an $L$ bank receives a call is equal to the fraction of single $S$ banks placing a call: $N_{S}$. Also, the probability of a single $S$ bank contacting a single $L$ bank is just equal to the fraction of single $L$ banks: $N_{L}$.

In sub-period 3, all banks choose whether to access and trade in the core market for reserves. An $S$ bank pays a cost $\gamma$ to access this market, whether it is single or not. It is free for $L$ banks to access this market and they always will. To derive closed-form solutions, we model this market as a centralized market in which participants take the core market rate $i_{m}$ as given. In section 6 , we describe how the results change when this market is a bilateral over-the-counter market (and present the details in our companion paper).

In sub-period 4, banks that still carry a reserve deficit after interbank market trades will have to borrow their deficit at the lending facility and suffer the penalty rate $i_{\ell}$. Banks that enjoy a reserve surplus after the interbank market trades will see it remunerated at the rate $i_{d}$ by the central bank. We refer to $i_{\ell}$ as the lending rate

\footnotetext{
${ }^{14}$ In our companion paper, we briefly discuss an extension in which single banks can also trade in sub-period 2.
} 
and to $i_{d}$ as the deposit rate, where $i_{d}<i_{\ell}$. The central bank sets three policy rates $\bar{i}, i_{d}$, and $i_{\ell}$ with the reserve balances $\bar{R}$, core market rates $i_{m}$ and the distribution of relationship rates $\hat{i}$ determined endogenously.

\begin{tabular}{|c|l|}
\hline Subperiod & Events \\
\hline \hline & Settlement \\
\hline 1 & Central bank tender \\
\hline 2 & Liquidity shocks, peripheral market trades, and relationship calls \\
\hline 3 & Core market trades \\
\hline 4 & Access to central bank facilities \\
\hline
\end{tabular}

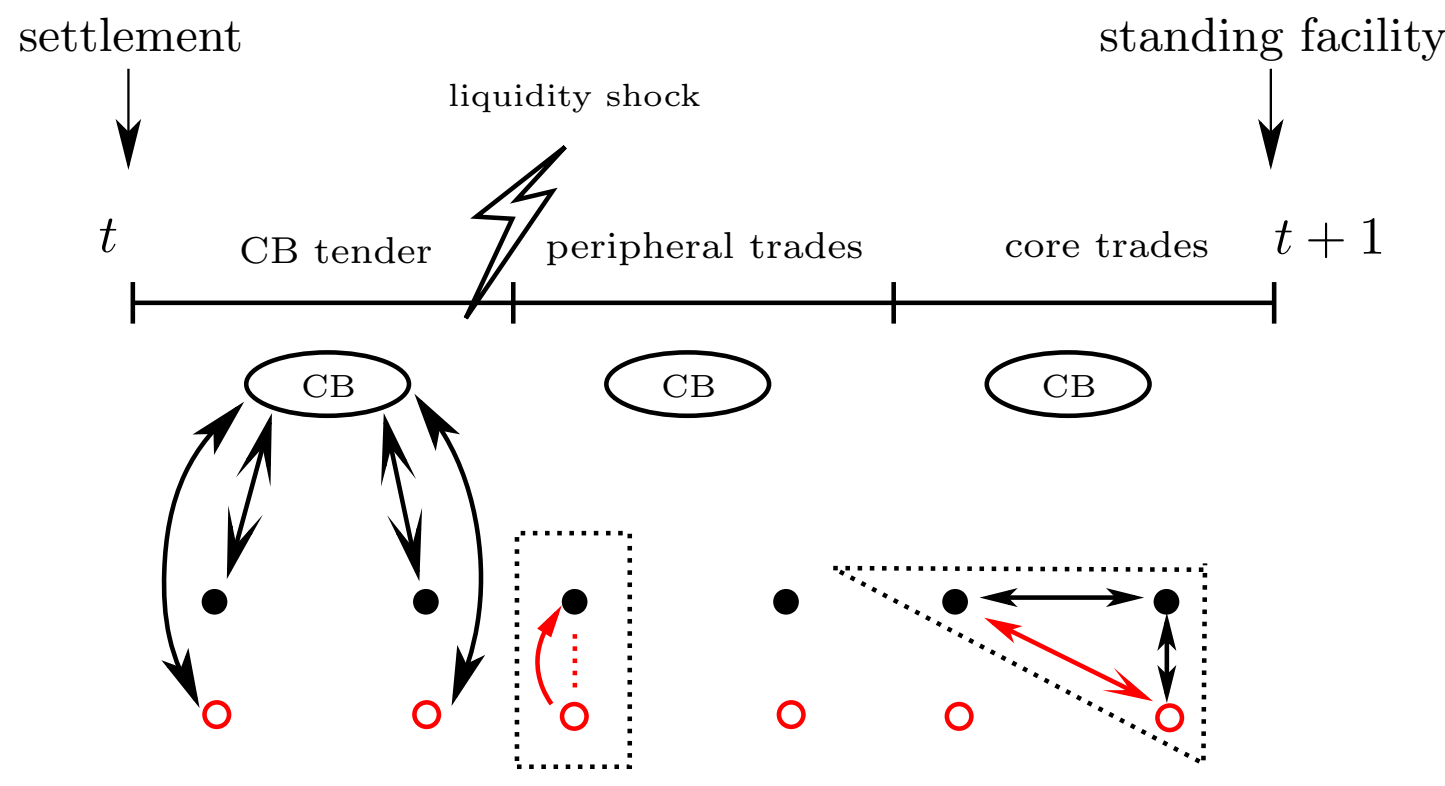

Figure 3: Timeline

\section{$3 \quad$ Equilibrium}

In this section, we set up the decision problem of each bank in each market and we define our equilibrium. We first define the payoff of each bank at the settlement stage, and then proceed backward to define the payoff in each of the preceding markets. 


\subsection{Sub-period 4. Central bank facilities and next-day set- tlement}

Consider a bank holding reserves $R$, with aggregate dues from previous trade $D$ (where $D>0$ would be an aggregate credit position while $D<0$ would represent an aggregate debit position) and relationship $n \in\{0,1\}$ where 0 stands for being a single bank. At the end of the day, a bank holding $R<0$ has to borrow $-R$ units of reserves from the central bank and pays an interest $i_{\ell}$ on this loan. Otherwise, this bank deposits $R>0$ with the central bank to earn $i_{d}$ on this amount. In the following settlement stage, the central bank collects or pays the amount of the numeraire goods corresponding to these loans and deposits. Also, banks settle their aggregate position with other institutions by producing and transferring the numeraire goods. If their aggregate position is positive, they end up consuming some of the numeraire. Therefore, the value of an $L$ bank just before accessing the central bank facilities is $V_{4}$, given by

$$
V_{4}(R, D, n)=\beta\{D+R[1+i(R)]\}+\beta \bar{V}_{1}(n)
$$

where

$$
i(R)=\left\{\begin{array}{ll}
i_{d} & \text { if } R \geq 0 \\
i_{\ell} & \text { if } R<0
\end{array},\right.
$$

and $\bar{V}_{1}(n)$ denotes the expected value of an $L$ bank with status $n \in\{0,1\}$ before the separation shock at the beginning of a period. Similarly, the value of an $S$ bank before accessing the central bank facilities is $v_{4}$, given by

$$
v_{4}(R, D, n)=\beta\{D+R[1+i(R)]\}+\beta \bar{v}_{1}(n),
$$

where $\bar{v}_{1}(n)$ denotes the value of an $S$ bank with status $n \in\{0,1\}$ before the separation shock. At the beginning of sub-period 1, a fraction $\sigma$ of existing relationships are broken up exogenously and a number of banks are without a relationship. Then 
the value functions of the different types of banks are,

$$
\begin{aligned}
& \bar{v}_{1}(n)=[1-\sigma(n)] v_{1}(1)+\sigma(n) v_{1}(0), \\
& \bar{V}_{1}(n)=[1-\sigma(n)] V_{1}(1)+\sigma(n) V_{1}(0),
\end{aligned}
$$

where $\sigma(0)=1, \sigma(1)=\sigma$, and $v_{1}(n), V_{1}(n)$ denote the expected values in sub-period 1.

\subsection{Sub-period 3. The core interbank market}

Before the standing facilities, there is the competitive core interbank market in which banks trade reserves among themselves. We denote by $\Omega^{+}($.$) the cumulative distri-$ bution of positive reserve holdings in the core market, so that $\Omega^{+}(\tilde{R})$ is the measure of banks with reserves $R \in[0, \tilde{R}]$. Similarly, we denote by $\Omega^{-}($.$) the cdf for negative$ reserve holdings in the core market, so that $\Omega^{-}(\tilde{R})$ is the measure of banks with reserves $R \in(-\infty, \tilde{R}]$. Therefore the aggregate amount of reserves in the core market is

$$
\bar{R} \equiv \int_{-\infty}^{0} R d \Omega^{-}(R)+\int_{0}^{\infty} R d \Omega^{+}(R)
$$

The competitive core market rate will then be ${ }^{15}$

$$
i_{m}(\bar{R})= \begin{cases}i_{\ell} & \text { if } \bar{R}<0 \\ \in\left[i_{d}, i_{\ell}\right] & \text { if } \bar{R}=0 \\ i_{d} & \text { if } \bar{R}>0\end{cases}
$$

We can now derive the value of $L$ banks at the beginning of the core market. For later use, we define the expected gains from trade for a borrower or a lender in the core market, respectively,

$$
\Pi(R)= \begin{cases}\beta R\left(i_{m}(\bar{R})-i_{\ell}\right) & \text { if } R \leq 0 \\ \beta R\left(i_{m}(\bar{R})-i_{d}\right) & \text { if } R>0 .\end{cases}
$$

\footnotetext{
${ }^{15}$ This bang-bang interest rate response to the aggregate reserve supply is simple but rather extreme. Section 6 extends the model to study an OTC core market and obtains a distribution of core rates that adjust smoothly to the reserve supply.
} 
Here, a bank with negative balances borrows from the core market at $i_{m}$ and avoids borrowing from the central bank facility at the lending rate $i_{\ell}$. Similarly, a bank with $R>0$ lends to the core market at $i_{m}$ instead of earning the deposit rate $i_{d}$. Then the value of one $L$ bank with $R$ units of reserves, aggregate dues $D$, and relationship status $n$ at the start of the core market is its no-trade payoff augmented with its expected gains from trade:

$$
V_{3}(R, D, n)=\Pi(R)+\beta\{D+R(1+i(R))\}+\beta \bar{V}_{1}(n)
$$

or

$$
V_{3}(R, D, n)=\beta R\left(1+i_{m}(\bar{R})\right)+\beta D+\beta \bar{V}_{1}(n)
$$

Obviously $V_{3}(R, D, n)$ is strictly increasing in $R$ with marginal value of reserve $\beta(1+$ $\left.i_{m}(\bar{R})\right)$.

We now turn to the value of $S$ banks at the start of the core market. The cost $\gamma$ of entering the core interbank market may be too prohibitive for some $S$ banks and hence an $S$ bank only participates in the core market when the gains from trading $\Pi(R)$ given by (6) compensate for the entry $\operatorname{cost} \gamma$. Therefore, the value function of an $S$ bank at the time of choosing whether to participate or not, given $R$, is ${ }^{16}$

$$
v_{3}(R, D, n)=\max \{\Pi(R)-\gamma, 0\}+\beta\{D+R(1+i(R))\}+\beta \bar{v}_{1}(n) .
$$

and using (6) a bank $S$ enters the core market if and only if

$$
\beta R\left(i_{m}(\bar{R})-i(R)\right)>\gamma
$$

So we obtain

\footnotetext{
${ }^{16}$ Note that $v_{3}$ is increasing in $R$ with marginal value $\beta[1+i(R)]$ if the bank does not enter the market, and with marginal value $\beta\left[1+i_{m}(\bar{R})\right]$ otherwise.
} 
Lemma 1. An $S$ bank enters the core market to borrow iff $R \leq \hat{R}^{-}$and enters to lend iff $R \geq \hat{R}^{+}$, with $\Pi\left(\hat{R}^{-}\right)=\Pi\left(\hat{R}^{+}\right)=\gamma$ and

$$
\begin{aligned}
\hat{R}^{-} & =\frac{-\gamma}{\beta\left[i_{\ell}-i_{m}(\bar{R})\right]}, \\
\hat{R}^{+} & =\frac{\gamma}{\beta\left[i_{m}(\bar{R})-i_{d}\right]} .
\end{aligned}
$$

Intuitively, an $S$ bank enters the core market only when it is worth paying the entry cost. This is the case only if the bank's reserve balance differs significantly from the required level 0 . Notice that the entry decision does not depend on the $S$ bank's relationship status, but only on its reserve holdings. Also, note that the core interbank rate never falls outside the corridor. We now move on to the (relationship) trades that occur in the periphery of the core market.

\subsection{Sub-period 2. Peripheral trades}

In sub-period 2, banks in a pre-established relationship trade their reserves, taking into account that the core market will open next. Single banks are inactive and their payoffs are given by (7) and (8) with $n=0$. Now consider two banks in a relationship where the $S$ bank holds $R_{S}$ while the $L$ bank holds $R_{L}$. We assume that both banks bargain to determine the loan amount $z$ from the $S$ bank to the $L$ bank as well as the loan rate $\hat{i}{ }^{17}$ In addition we assume proportional bargaining with weight $\theta$ assigned to $S$ banks.

Obviously, the bargaining outcome depends on the banks' outside option. If bank $L$ has been contacted by a single $S$ bank, we assume its threat point with the incumbent is no trade and breaking up its current relationship. In case of breakup, the incumbent $S$ bank's status drops to $n=0$, while the $L$ bank gets in a relationship with the new $S$ bank, and so its status remains $n=1$. If bank $L$ has

\footnotetext{
${ }^{17}$ We use the convention that bank $S$ lends to banks $L$ whenever $z>0$ and borrows from $L$ whenever $z<0$.
} 
not been contacted, then the threat point is no trade, but keeping the relationship into the next period so that $n=1$ for both banks. ${ }^{18}$

From (7) and (8) we can find the trading surplus for an $S$ and an $L$ bank as a function of the terms of trade, respectively $T S_{S}(z, \hat{i} ; c)$ and $T S_{L}(z, \hat{i} ; c)$, where $c \in\{0,1\}$ indicates whether the $L$ bank has been contacted by another $S$ bank $(c=1)$ or not $(c=0) \cdot{ }^{19}$ Then the bargaining problem is

$$
\max _{z, \hat{i}} T S_{S}(z, \hat{i} ; c)
$$

subject to

$$
T S_{L}(z, \hat{i} ; c)=(1-\theta)\left[T S_{S}(z, \hat{i} ; c)+T S_{L}(z, \hat{i} ; c)\right]
$$

We assume that while banks can promise to repay loans the next day, they can neither commit to trade in the future nor to the terms of trade in future meetings. For simplicity, we focus on period-by-period bargaining, with the solution given by the following proposition. ${ }^{20}$

Proposition 2. (Tiering structure) The size of the relationship loan is $R_{S}$, the reserves holdings of the $S$ bank. $S$ banks with a relationship never enter the core market.

This result is intuitive. As derived in the Appendix, the loan size $z$ is chosen to maximize the total surplus, given the banks's reserves, while the rate $\hat{i}$ is chosen to split the surplus according to the surplus-sharing protocol. Since bank $L$ can access the core market at no cost, it will provide intermediation by absorbing all the (excess) reserves of bank $S$ (positive or negative) while bank $S$ can avoid the entry

\footnotetext{
${ }^{18}$ These threat points are subgame-perfect.

${ }^{19}$ The details are in the Appendix.

${ }^{20}$ Given reserves holdings, the terms of trades under full or limited commitment are the same. However, reserve holdings can differ with the degree of commitment for a simple reason: In the absence of commitment, banks internalize the fact that they can be cornered by their trading partner, which induces them to adopt a suboptimal bidding strategy at the central bank tender.
} 
cost. $^{21}$ The relationship rate is the price bank $S$ pays to bank $L$ to intermediate the access to the core market. Using the solution to the bargaining problem above, it is easy to see that the relationship rate splits the gains from trade according to the bargaining power. The gains from trade are made of three components: First, bank $S^{\prime}$ opportunity cost of using bank $L$ as an intermediary, i.e.

$$
\max \left\{\beta\left[1+i\left(R_{S}\right)\right] R_{S},-\gamma+\beta\left[1+i_{m}(\bar{R})\right] R_{S}\right\}
$$

Second, bank $L$ 's benefit of intermediating bank $S$ ' balances

$$
\left[1+i_{m}(\bar{R})\right] R_{S}
$$

Third, bank $S$ 's willingness to pay to retain the relationship with bank $L$, whenever it is contacted by another bank, i.e.

$$
(1-\sigma) v_{1}(1)+\sigma v_{1}(0)-v_{1}(0)
$$

Defining

$$
\mathcal{V} \equiv-(1-\sigma)\left[v_{1}(1)-v_{1}(0)\right]
$$

as the cost for bank $S$ of giving up the relationship, we can then write the relationship rate from the bargaining problem as

$$
\begin{aligned}
1+\hat{i}\left(R_{S}, R_{L}, c\right)= & (1-\theta) \frac{\max \left\{\beta\left[1+i\left(R_{S}\right)\right] R_{S},-\gamma+\beta\left[1+i_{m}(\bar{R})\right] R_{S}\right\}}{\beta R_{S}} \\
& +\theta\left[1+i_{m}(\bar{R})\right]+(1-\theta) \frac{c \mathcal{V}}{R_{S}}
\end{aligned}
$$

where $c=1$ if bank $L$ was contacted, and $c=0$ otherwise. Using (6) and the entry decision of single banks we obtain

$\hat{i}\left(R_{S}, R_{L}, c\right)= \begin{cases}\theta i_{m}(\bar{R})+(1-\theta) i\left(R_{S}\right)+(1-\theta) \frac{\mathcal{V}}{R_{S}}, & \text { if no entry when single } \\ \theta i_{m}(\bar{R})+(1-\theta)\left[i_{m}(\bar{R})-\frac{\gamma}{\beta R_{S}}\right]+(1-\theta) \frac{\mathcal{V}}{R_{S}}, & \text { if entry when single }\end{cases}$

\footnotetext{
${ }^{21}$ The centralized structure of the core market guarantees that the $L$ bank can always trade. Hence, there is no need for $S$ banks in a relationship to enter the core market. When the core market is OTC instead of centralized, $S$ banks in a relationship may still enter the market to insure against the event that the $L$ bank would not find a proper match.
} 
Notice that the relationship rate is only a function of $R_{S}$, and that the rate with no entry is always smaller than the rate with entry. The relationship rate is a sum of two parts. The first part is a weighted sum of the $L$ bank's benefit of obtaining $R_{S}$ and the $S$ bank's opportunity cost of giving up $R_{S}$. This part is always between $i_{d}$ and $i_{\ell}$. The second part $\mathcal{V} / R_{S}$ is the $S$ bank's cost of giving up the relationship. This part can be positive or negative depending on whether the $S$ bank is a borrower or a lender. Comparing the relationship rate and the core rate, we can define a relationship premium as follows.

Lemma 3. The relationship rate is $\hat{i}\left(R_{S}, c\right)=i_{m}(\bar{R})-P\left(R_{S}\right)$ where $P\left(R_{S}\right)$ is the relationship premium defined as,

$$
P\left(R_{S}\right)=(1-\theta)\left[\min \left\{i_{m}(\bar{R})-i\left(R_{S}\right), \frac{\gamma}{\beta R_{S}}\right\}-\frac{c \mathcal{V}}{R_{S}}\right],
$$

with $P\left(R_{S}\right)>0$ for $R_{S}>0$ and $P\left(R_{S}\right)<0$ for $R_{S}<0$.

The relationship premium captures how much relationship rates $\hat{i}$ of peripheral trades deviate from the core rate $i_{m}$. When $S$ banks do not have full bargaining power (i.e. $\theta<1$ ), the premium is non-zero when the $S$ banks value the relationship (i.e. $\left.\mathcal{V}=-(1-\sigma)\left[v_{1}(1)-v_{1}(0)\right]<0\right)$ and the $L$ bank can immediately rebuild a new one (i.e. $c=1$ ). Also, the premium is non-zero when it is costly to enter the core market (i.e. $\gamma>0)$. Notice that whenever the $S$ bank is a lender $\left(R_{S}>0\right), \mathcal{V} / R_{S}$ is negative as long as the $S$ bank values the relationship. In this case, the relationship rate is driven down. Similarly, whenever the $S$ bank is a borrower $\left(R_{S}<0\right)$ the relationship rate is raised as the $S$ bank values the relationship. ${ }^{22}$ In addition, when $\mathcal{V}=0$, the relationship rate always stays within the corridor. Otherwise, the relationship rate can fall below $i_{d}$ or go above $i_{\ell}$. We formalize this result in the following corollary,

\footnotetext{
${ }^{22}$ Interestingly, this is consistent with the finding by Ashcraft and Duffie (2007) that "[t]he rate negotiated is higher for lenders who are more active in the federal funds market relative to the borrower. Likewise, if the borrower is more active in the market than the lender, the rate negotiated is lower, other things equal."
} 
Corollary 1. Relationship rates for peripheral trades may fall outside of the corridor when the $L$ bank is contacted by an $S$ bank: With $R_{S}>0, \hat{i}\left(R_{S}\right)<i_{d}$ iff

$$
i_{m}(\bar{R})<i_{d}-\frac{(1-\theta) \mathcal{V}}{\theta R_{S}} .
$$

With $R_{S}<0, \hat{i}\left(R_{S}\right)>i_{\ell}$ iff

$$
i_{m}(\bar{R})>i_{\ell}-\frac{(1-\theta) \mathcal{V}}{\theta R_{S}} .
$$

This concludes the analysis of trades between two banks in a relationship. To summarize, $S$ banks use the $L$ bank as an intermediary to access the interbank market and may even agree to pay a premium to continue the relationship. So the relationship rate could well hover above $i_{\ell}$ whenever the $S$ bank is borrowing from the $L$ bank, or below $i_{d}$ whenever it is lending to $L$.

\subsection{Sub-period 2. Relationship-building calls}

Before peripheral trades, single $S$ banks can pay a cost $\kappa$ to contact a bank $L$. At this stage, all single $S$ banks are the same, so either they all pay the cost, or none of them do, or they use a mixed strategy if they are indifferent.

To be precise, at the start of sub-period 2, a fraction $1-N_{L}$ of $L$ banks are in a relationship and a fraction $N_{L}$ is single. Therefore, a single $S$ bank searching contacts a single $L$ with probability $N_{L}$. Also an $L$ bank expects a contact with probability $N_{S}$, where $N_{S}$ is the fraction of $S$ banks that are single and searching.

The fraction of $L$ (and $S$ ) banks in a relationship is $1-N_{L}$. So at the beginning of sub-period 2 the fraction of $L$ banks that are single are all but ( $a$ ) those who were in a relationship last period and the relationship was not severed, and (b) those who were not in a relationship last period, got a contact and the new relationship was not severed:

$$
N_{L}=1-[\underbrace{\left(1-N_{L}\right)(1-\sigma)}_{(a)}+\underbrace{N_{L} N_{S}(1-\sigma)}_{(b)}],
$$


or

$$
N_{L}=\frac{\sigma}{(1-\sigma) N_{S}+\sigma}
$$

$S$ banks search whenever the expected benefit is higher than the search cost $\kappa$. Hence, the fraction of $S$ banks searching is

$$
N_{S}= \begin{cases}N_{L} & \text { if } N_{L}(1-\sigma) \beta\left[v_{1}(1)-v_{1}(0)\right]>\kappa \\ 0 & \text { if } N_{L}(1-\sigma) \beta\left[v_{1}(1)-v_{1}(0)\right]<\kappa \\ {\left[0, N_{L}\right]} & \text { otherwise }\end{cases}
$$

The left-hand side of the above inequality condition captures that a single $S$ bank successfully finds a single $L$ bank with probability $N_{L}$, in which case a new relationship is formed but only survived with probability $1-\sigma$, in which case it brings a surplus $v_{1}(1)-v_{1}(0)$ from the next period onward.

\subsection{Sub-period 1. Central bank tender}

We now define the expected payoffs of different types of banks at the end of subperiod 1. We use $m_{n}$ and $M_{n}$ to denote, respectively, the reserve balances of $S$ and $L$ banks with relationship status $n \in\{0,1\}$ when they exit the central bank tender but before they know their liquidity shock. Define $\mathbf{M}=\left(M_{0}, M_{1}, m_{0}, m_{1}\right)$ and let the dues from the relationship loan be $D\left(R_{S}, c\right)$, where again $c=1$ if bank $L$ has been contacted by another $S$ bank, and $c=0$ otherwise, i.e. $D\left(R_{S}, c\right)=\left[1+\hat{i}\left(R_{S}, c\right)\right] R_{S}$. Then the expected value of a matched $L$ bank with reserve holdings $M$ before the shock is

$V_{2}(M, 1 ; \mathbf{M})=\int_{-\infty}^{\infty} \int_{-\infty}^{\infty} E_{c}\left[V_{3}\left(M+\varepsilon+m_{1}+\xi,-D\left(m_{1}+\xi, c\right), 1\right)\right] d G(\varepsilon) d F(\xi)+\beta \bar{V}_{1}(1)$

and in the Appendix, we show that bank L's marginal benefit of an extra dollar is just the discounted benefit of lending this extra dollar in the core market, i.e.

$$
\frac{\partial V_{2}(M, 1 ; \mathbf{M})}{\partial M}=\beta\left(1+i_{m}(\bar{R})\right)
$$


Similarly, the expected value of a bank $S$ in a relationship holding reserves $m_{1}$ before the shock is

$$
v_{2}\left(m_{1}, 1 ; \mathbf{M}\right)=\int_{-\infty}^{\infty} E_{c}\left[v_{3}\left(0, D\left(m_{1}+\xi, c\right), 1\right)\right] d F(\xi)+\beta \bar{v}_{1}(1)
$$

When choosing $m_{1}$, the $S$ bank takes into account how it affects the probability of accessing the core market. In the Appendix, we show that the value of the marginal dollar is

$$
\begin{aligned}
\frac{\partial v_{2}\left(m_{1}, 1 ; \mathbf{M}\right)}{\partial m_{1}}= & \beta\left(1+i_{m}(\bar{R})\right)+\beta(1-\theta)\left[i_{d}-i_{m}(\bar{R})\right]\left[F\left(\hat{R}^{+}-m_{1}\right)-F\left(-m_{1}\right)\right] \\
& +\beta(1-\theta)\left[i_{\ell}-i_{m}(\bar{R})\right]\left[F\left(-m_{1}\right)-F\left(\hat{R}^{-}-m_{1}\right)\right]
\end{aligned}
$$

where $\hat{R}^{+}$and $\hat{R}^{-}$are given by (9). The first term is the discounted benefit of lending this extra dollar in the core market when the $S$ bank enters the core market. The second and third terms capture the additional values when the $S$ bank passes the core market to use the central bank facilities. Notice that if $i_{m}(\bar{R})=\left(i_{\ell}+i_{d}\right) / 2$ then $\partial v_{2}\left(m_{1}, 1 ; \mathbf{M}\right) / \partial m_{1}=\beta\left(1+i_{m}(\bar{R})\right)$.

The expected value of a single $L$ bank holding $M$ units of reserves before the shock is $V_{2}(M, 0 ; \mathbf{M})$ defined in a similar way as above, and the value of an extra dollar for a single $L$ bank is also the discounted value of lending this extra dollar in the core market:

$$
\frac{\partial V_{2}(M, 0 ; \mathbf{M})}{\partial M}=\beta\left(1+i_{m}(\bar{R})\right) .
$$

Finally, the expected value of a single $S$ bank holding reserves $m$ before the shock, is $v_{2}\left(m_{0}, 0 ; \mathbf{M}\right)$ defined as above, and the value of an extra dollar for this bank is

$$
\begin{aligned}
\frac{\partial v_{2}\left(m_{0}, 0 ; \mathbf{M}\right)}{\partial m_{0}}= & \beta\left(1+i_{m}(\bar{R})\right)+\beta\left[i_{d}-i_{m}(\bar{R})\right]\left[F\left(\hat{R}^{+}-m_{0}\right)-F\left(-m_{0}\right)\right] \\
& +\beta\left[i_{\ell}-i_{m}(\bar{R})\right]\left[F\left(-m_{0}\right)-F\left(\hat{R}^{-}-m_{0}\right)\right] .
\end{aligned}
$$

Notice that relative to (15), the single $S$ bank fully internalizes the benefit/cost of this extra dollar, as it does not have to share it with an $L$ bank. 
In sub-period 1, the central bank offers to sell and buy reserves at a rate $\bar{i}^{23}$ This "tender" consists of one-period loans of reserve balances, which will be paid back in the next settlement stage. Expecting that other banks will bid $\mathbf{M}$, the decision of an $L$ bank with $n \in\{0,1\}$ is:

$$
V_{1}(n)=\max _{M_{n}}-\beta(1+\bar{i}) M_{n}+V_{2}\left(M_{n}, n ; \mathbf{M}\right)
$$

and

$$
v_{1}(n)=\max _{m_{n}}-\beta(1+\bar{i}) m_{n}+v_{2}\left(m_{n}, n ; \mathbf{M}\right) .
$$

The first-order conditions for $L$ banks are

$$
\frac{\partial V_{2}\left(M_{n}, n ; \mathbf{M}\right)}{\partial M_{n}}=\beta(1+\bar{i}),
$$

and similarly for $S$ banks,

$$
\frac{\partial v_{2}\left(m_{n}, n ; \mathbf{M}\right)}{\partial m_{n}}=\beta(1+\bar{i})
$$

So, we can find the bids of each bank using (14)-(17). In equilibrium the choice of reserves of individual banks should be consistent with M. Also, notice that if $\bar{i}>i_{\ell}$, then banks can earn infinite risk-free profit by holding an infinitely large negative amount of reserves; if $\bar{i}<i_{d}$, then banks can earn infinite risk-free profit by holding an infinitely large positive amount of reserves. Therefore, no arbitrage requires $\bar{i} \in\left[i_{d}, i_{\ell}\right]$.

Given $\mathbf{M}$, the excess reserves in the core market $\bar{R}$ are given by

$$
\bar{R}=\frac{1-N_{L}}{2} M_{0}+\frac{N_{L}}{2} M_{1}+\frac{1-N_{L}}{2} \int\left(m_{0}+\xi\right) \mathbb{I}_{e}\left(m_{0}+\xi\right) d F(\xi)+\frac{N_{L}}{2} m_{1}
$$

where $\mathbb{I}_{e}=1$ when a bank $S$ enters the core market and $\mathbb{I}_{e}=0$ when it does not. Since $L$ banks always enter the interbank market, there are $1-N_{L}$ single $L$ banks holding

\footnotetext{
${ }^{23} \mathrm{An}$ alternative view, which is more in line with some central banks' practice, is that the central bank supplies/purchases $M$ units of reserves and banks bid a schedule of rates and corresponding reserves amount they are willing to obtain at this rate. Market clearing implies $\sum_{n} M_{n}+m_{n}=M$, and the stop-out rate is $\bar{i}$, such that the first-order condition of each bank holds.
} 
$M_{0}$. But only those $1-N_{L}$ single $S$ banks that sustained a large enough shock $\xi$ will pay the entry cost. Finally, as $L$ banks in a relationship always intermediate entry in the centralized interbank market for $S$ banks, the average $L$ bank in a relationship enters the relationship market with $M_{1}+m_{1}$. From (5) and the above conditions, it is straightforward to show that

Proposition 4. $i_{m}(\bar{R})=\bar{i}$ and we only have three types of equilibrium (and policy rate):

$$
\bar{i}= \begin{cases}i_{\ell} & , m_{n}=-\infty \text { and } M_{n} \text { such that } \bar{R}<0 \\ \frac{i_{\ell}+i_{d}}{2} & , m_{n}=0 \text { and } M_{n} \text { such that } \bar{R}=0 \\ i_{d} & , m_{n}=\infty \text { and } M_{n} \text { such that } \bar{R}>0\end{cases}
$$

Also, $N_{S}>0$ (i.e. $S$ banks search) only if $\bar{i}=\left(i_{\ell}+i_{d}\right) / 2$.

This means that only the policy rate set at the midpoint of the corridor will induce relationship formation. This is due to the centralized structure of the core market. As we show in our companion paper, it is possible to eliminate the extreme cases by extending the model to have a core market with an OTC instead of a centralized structure.

\section{Characterization of the equilibrium}

We can now define an equilibrium. Recall that $\mathbf{M}=\left\{M_{n}, m_{n}\right\}_{n=0,1}$. Then,

Definition 5. Given $\bar{i} \in\left\{i_{d}, \frac{i_{\ell}+i_{d}}{2}, i_{\ell}\right\}$ a steady-state equilibrium is a list $\left\{N_{S}, N_{L}, \mathbf{M}\right\}$ consisting of the fraction $N_{S}$ of single $S$ banks searching, the fraction $N_{L}$ of single $L$ banks, and the banks' money demand $\mathbf{M}$, such that, given the policy rates $\bar{i}, i_{d}$, and $i_{\ell}$, the search decision by $S$ banks, reserves holdings, and entry by $S$ banks in the core market are optimal and consistent with the bargaining solutions.

Let

$$
\Gamma \equiv \int_{-\infty}^{\infty}\left\{\beta \theta\left[\mathbb{I}_{e} \frac{\gamma}{\beta}+\mathbb{I}_{n e}\left[\frac{i_{\ell}+i_{d}}{2}-i(\xi)\right] \xi\right]\right\} d F(\xi)
$$


be the expected per-period benefit of a relationship for the $S$ bank when the rate in the centralized market is at the midpoint of the corridor. Then we obtain the existence and uniqueness of the equilibrium.

Proposition 6. A unique equilibrium exists. When $\bar{i} \in\left\{i_{d}, i_{\ell}\right\}$ then $N_{S}=0$ and there is no relationship. When $\bar{i}=\left(i_{\ell}+i_{d}\right) / 2$ then there are three cases, with

$$
\bar{\kappa}=\frac{\beta(1-\sigma)}{[1-\beta(1-\sigma)]} \Gamma, \text { and } \underline{\kappa}=\frac{N_{L}^{*}(1-\sigma) \beta}{\left[1-\beta(1-\sigma)\left(1-N_{L}^{*}(1-\theta)\right)\right]} \Gamma,
$$

(1) When $\kappa<\underline{\kappa}$, all single $S$ banks search, $N_{S}=N_{L}^{*}=\frac{-\sigma+\sqrt{\sigma^{2}+4(1-\sigma) \sigma}}{2(1-\sigma)}$, and

$$
v_{1}(1)-v_{1}(0)=\frac{\Gamma+\kappa}{1-\beta(1-\sigma)\left(1-N_{L}^{*}-N_{S}^{*}(1-\theta)\right)} .
$$

(2) When $\kappa \in[\underline{\kappa}, \bar{\kappa}], S$ banks use a mixed strategy and $N_{S}=\bar{N}_{S}$ given by (C.1), and

$$
v_{1}(1)-v_{1}(0)=\frac{\Gamma}{1-\beta(1-\sigma)\left[1-\bar{N}_{S}(1-\theta)\right]} .
$$

(3) When $\kappa>\bar{\kappa}$, $S$ banks do not search, $N_{S}=0$, and

$$
v_{1}(1)-v_{1}(0)=\frac{\Gamma}{1-\beta(1-\sigma)} .
$$

Proof. We relegate the proof to the Appendix.

Intuitively, when $\kappa$ is low, all single $S$ banks will try to build a relationship. When $\kappa$ is high, there is no relationship lending because it is too costly for $S$ banks to build one. For the intermediate level of $\kappa$, single $S$ banks are indifferent between building a relationship and staying single.

\section{Qualitative implications}

This section derives some qualitative implications of the benchmark model. Specifically, we study (i) how policy and other parameters affect the network structure of 
the interbank market, (ii) why interbank rates can go below the floor in the short run and in the long run, (iii) how the interbank market will react dynamically to an exit from a floor system, and (iv) how regulation on exposure limits can affect the interbank market.

\subsection{Interbank network}

To study the network structure of interbank trades, we first derive some comparative statics on the entry decision of single banks in the core market. Recall that the entry decision by single $S$ banks is given by the two thresholds defined in (9). Since $i_{m} \in\left\{i_{d},\left(i_{\ell}+i_{d}\right) / 2, i_{\ell}\right\}$, these thresholds only depend on the corridor size $i_{\ell}-i_{d}$. As a consequence the relationship benefits $\Gamma$ also depend on the corridor size $i_{\ell}-i_{d}$.

Lemma 7 . When $\bar{i}=\left(i_{\ell}+i_{d}\right) / 2$, the per-period gains from a relationship $\Gamma$ is increasing in $i_{\ell}-i_{d}, \theta$, and the variance of the shock $\xi$. Also $\Gamma \rightarrow 0$ when $i_{\ell} \rightarrow i_{d}$.

As a relationship provides intermediation to the core market, the expected benefit of a relationship for the $S$ banks increases when the corridor is wider, their bargaining power is higher and the liquidity shock is more uncertain. ${ }^{24}$ As a consequence, $S$ banks' incentives to search and build relationships are affected by the same set of variables.

Lemma 8. $\underline{\kappa}, \bar{\kappa}$ and $\bar{N}_{S}$ are increasing in $i_{\ell}-i_{d}, \theta$, and the variance of the shock $\xi$, and $\bar{N}_{S}$ is decreasing in $\kappa . N_{S} \rightarrow 0$ as $i_{\ell} \rightarrow i_{d}$.

In words, single $S$ banks search more actively when the gains from relationship lending are larger (that is, when the corridor is larger), when they have more bargaining power, or when the variance of the shock is higher. Naturally, they search less when the cost of searching is higher. Finally, single $S$ banks do not search if $i_{\ell}=i_{d}$, as in this case there is no gain from trade.

\footnotetext{
${ }^{24}$ If $2>\frac{f(x) x}{1-F(x)}$ for all $x>0$ then $\Gamma$ is also increasing in $\gamma$.
} 
After examining the trades among banks, we now study the usage of standing facilities and derive the amount borrowed from or deposited at the standing facility. Access to the two standing facilities is determined by the amount of excess reserves in the centralized market $\bar{R}$ together with the usage of the facilities by single $S$ banks that do not enter the core market. So, access to the lending facility when the policy rate is at the midpoint of the corridor (i.e. $\bar{R}=0$ ) is

$$
\frac{1}{2} \frac{\sigma}{(1-\sigma) N_{S}+\sigma} \int_{\hat{R}^{-}}^{-m_{0}}\left(m_{0}+\xi\right) d F(\xi)=\frac{1}{2} \frac{\sigma}{(1-\sigma) N_{S}+\sigma} \int_{\hat{R}^{-}}^{0} \xi d F(\xi)
$$

The integrand is decreasing as $i_{\ell}-i_{d}$ increases, and $N_{S}$ is either constant or increasing: As it is more costly to stay out of the core market, single banks tend to search more and enter the core market more frequently. As a result, there is a lower access to the lending facility (and also for the deposit facility). ${ }^{25}$

Finally, we study how the corridor width affects the network size. Recall that Beltran, Bolotny, and Klee (2015) show a decline in the number of loans from peripheral banks to core banks as the Fed reduced the corridor size. Hence, we should expect a positive relationship between the width of the corridor and the number of relationship loans. In our model, the size of the peripheral network $\mathcal{P}$ is defined by the measure of banks in a relationship. This is $1-N_{L}$, or

$$
\mathcal{P}=1-\frac{\sigma}{(1-\sigma) N_{S}+\sigma}
$$

which is positively correlated with $N_{S}$. Hence, as the corridor increases, more $S$ banks search and the size of the peripheral network $\mathcal{P}$ increases, as in the data.

The size of the core network $\mathcal{C}$ is defined by $1 / 2$ measure of $L$ banks as well as

\footnotetext{
${ }^{25}$ Access to the deposit facility is
}

$$
\frac{1}{2} \frac{\sigma}{(1-\sigma) N_{S}+\sigma} \int_{-m_{0}}^{\hat{R}^{+}}\left(m_{0}+\xi\right) d F(\xi) .
$$


single $S$ banks that enter the core market, or

$$
\begin{aligned}
\mathcal{C} & =\frac{1}{2}+\frac{1}{2} N_{L}\left[1-F\left(\hat{R}^{+}\right)+F\left(\hat{R}^{-}\right)\right] \\
& =\frac{1}{2}+\frac{1}{2} \frac{\sigma}{(1-\sigma) N_{S}+\sigma}\left[1-F\left(\hat{R}^{+}\right)+F\left(\hat{R}^{-}\right)\right] .
\end{aligned}
$$

As the corridor increases, there are two counteracting effects: (1) more $S$ banks are in a relationship, which decreases $\mathcal{C}$, but (2) those $S$ banks with no relationship enter the core market more often, thus increasing $\mathcal{C}$. So the effect of an increasing corridor on the size of the network in the core market $\mathcal{C}$ is indeterminate, but the volume of trade in the core market will increase. Finally, single and inactive $S$ banks always access the standing facilities and there is a fraction $\mathcal{I}$ of such banks,

$$
\mathcal{I}=\frac{1}{2} \frac{\sigma}{(1-\sigma) N_{S}+\sigma}\left[F\left(\hat{R}^{+}\right)-F\left(\hat{R}^{-}\right)\right] .
$$

As for $\mathcal{C}$, the effect of an increase in the corridor on $\mathcal{I}$ is indeterminate.

Finally, we used the labels $L$ and $S$ as standing for large and small. Even when $F=G$ and there is no difference between these two banks ex ante, aside from their access to the core market, $L$ and $S$ banks will tend to look large and small in equilibrium. Indeed, when they are matched, $S$ banks will always use the intermediation provided by the $L$ bank to indirectly access the core market. As a result, the $L$ bank will trade more often and larger amounts than $S$ banks.

\subsection{Trades below the floor}

As discussed in the introduction, at times, banks have been trading outside of the corridor, even though they had access to the central bank standing facilities at no cost, especially when there is excess liquidity in the banking system. How can our model match this fact? We will provide conditions under which the interbank rate may fall below the floor of the corridor $i_{d}$ both in the steady state and in response to a temporary liquidity shock. 


\section{(i) Steady state}

Below, we show that $S$ banks with a relatively small $\xi>0$ will trade below the floor whenever their $L$ bank partner has been contacted by a single $S$ bank. We already showed that when $N_{S}>0$, the relationship loans rate may fall outside of the corridor, i.e. $\hat{i}\left(R_{S}\right)<i_{d}$ if and only if $m_{1}+\xi>0$ and

$$
i_{m}(\bar{R})<i_{d}-\frac{(1-\theta) \mathcal{V}}{\theta\left(m_{1}+\xi\right)},
$$

or $\hat{i}\left(R_{S}\right)>i_{\ell}$ if and only if $m_{1}+\xi<0$ and

$$
i_{m}(\bar{R})>i_{\ell}-\frac{(1-\theta) \mathcal{V}}{\theta\left(m_{1}+\xi\right)} .
$$

Recall that there are no relationships except when the policy rate is at the midpoint of the corridor. In that case $m_{1}=0$ and $i_{m}(\bar{R})=\left(i_{\ell}+i_{d}\right) / 2$. Then the relationship rate falls below the floor whenever bank $S$ is holding $\xi>0$ relatively small, or ${ }^{26}$

$$
\frac{\left(i_{\ell}-i_{d}\right)}{2} \xi<\frac{(1-\theta)}{\theta}(1-\sigma)\left[v_{1}(1)-v_{1}(0)\right],
$$

where $v_{1}(1)-v_{1}(0)$ is given in Proposition 6. As $i_{\ell} \rightarrow i_{d}$, we have $\hat{R}^{+} \rightarrow \infty$ and $\hat{R}^{-} \rightarrow-\infty$, as well as

$$
\Gamma \rightarrow \beta \theta\left[\frac{i_{\ell}-i_{d}}{2}\right] 2 \int_{0}^{\infty} \xi d F(\xi) .
$$

While $\bar{N}_{S} \rightarrow 0$, all $S$ banks in a relationship receiving shock $\xi$ such that

$$
\xi<\frac{2(1-\theta) \beta(1-\sigma) \int_{0}^{\infty} \xi d F(\xi)}{1-\beta(1-\sigma)},
$$

\footnotetext{
${ }^{26}$ When $N_{S}=\bar{N}_{S}$, this expression becomes

$$
\xi<\frac{(1-\theta)}{\theta} \frac{(1-\sigma) \Gamma}{\left(\frac{i_{\ell}-i_{d}}{2}\right)\left[1-\beta(1-\sigma)\left[1-\bar{N}_{S}(1-\theta)\right]\right]},
$$

while when $N_{S}=N_{L}^{*}=\frac{-\sigma+\sqrt{\sigma^{2}+4(1-\sigma) \sigma}}{2(1-\sigma)}$ it is

$$
\xi<\frac{(1-\theta)(1-\sigma)}{\theta\left(\frac{i_{\ell}-i_{d}}{2}\right)} \frac{\Gamma+\kappa}{\left[1-\beta(1-\sigma)\left(1-N_{L}^{*}(2-\theta)\right)\right]} .
$$
}


will trade below the floor. That is, with a small corridor, while there are fewer relationships, it is more likely for a relationship trade to occur below the floor.

\section{(ii) Temporary liquidity shock}

We now consider a temporary liquidity shock to the interbank market. Consider a steady-state equilibrium in which the central bank sets $\bar{i}=\left(i_{\ell}+i_{d}\right) / 2$ and thus there are banks in active relationships. Suppose the central bank mistakenly allocates extra reserves so that there is a (small) amount of excess reserves in the core market, $\bar{R}>0$, only for the current period. ${ }^{27}$ Hence, the interbank rate falls to the floor $i_{m}(\bar{R})=i_{d}$. In addition, the central bank's mistake does not affect the search behavior of $S$ banks and thus $\mathcal{V}$ remains at its steady-state value, which is negative. From (11), we have

$$
\hat{i}\left(R_{S}, c\right)= \begin{cases}i_{d}+\frac{c(1-\theta) \mathcal{V}}{R_{S}}, & \text { if no entry when single } \\ i_{d}-(1-\theta) \frac{\gamma}{\beta R_{S}}+\frac{c(1-\theta) \mathcal{V}}{R_{S}}, & \text { if entry when single. }\end{cases}
$$

As a result, all relationship loans from an $S$ bank to an $L$ bank (i..e. $R_{S}>0$ ) that has a contact (i.e. $c=1$ ) are traded below the floor.

\subsection{Exit from a floor system}

In response to the recent financial crisis, many central banks have moved their monetary implementation framework from a symmetric corridor system to a floor system by significantly increasing reserve balances and setting the target rate at the bottom instead of the midpoint of the corridor. One natural question is how the interbank market will react when the central bank decides to exit from the floor system and return to the conventional corridor system. To answer this question, we derive the transition path as the interbank market converges from the old to the new steadystate equilibrium.

\footnotetext{
${ }^{27} \mathrm{An}$ alternative interpretation is that the central bank conducts a short-term intervention by injecting liquidity into the market with a commitment to withdraw it in the next period. See Berentsen and Waller (2011).
} 
Suppose the economy is initially in a steady state with $\bar{i}=i_{d}$. Then Proposition 6 implies that there is no relationship in equilibrium. Suppose the central bank announces at $T$ that it will set $\bar{R}=0$ or $\bar{i}=\left(i_{\ell}+i_{d}\right) / 2$ from the next period onward. Then the only changing variable in period $T$ is the search behavior of single $S$ banks. As there are no relationships when $\bar{i}=i_{d}$, all $S$ banks that search in period $T$ will find a match.

Recall that the fraction of $L$ (and $S$ ) banks in a relationship is $1-N_{L}(t)$ in period $t \geq T$, with $N_{L}(T)=1$. The fraction of single $L$ banks at $t+1$ are all $L$ banks but $(a)$ those who were in a relationship at $t$ that was not severed, and $(b)$ those who were not in a relationship at $t$, got a contact, and the new relationship was not severed:

$$
N_{L}(t+1)=1-\left[\left(1-N_{L}(t)\right)(1-\sigma)+N_{L}(t) N_{S}(t)(1-\sigma)\right]
$$

The fraction of $S$ banks searching in period $t$ is given by their entry decision when they benefit from the relationship from tomorrow onward,

$$
N_{S}(t)= \begin{cases}N_{L}(t) & \text { if } N_{L}(t)(1-\sigma) \beta\left[v_{1}(1, t+1)-v_{1}(0, t+1)\right]>\kappa \\ 0 & \text { if } N_{L}(t)(1-\sigma) \beta\left[v_{1}(1, t+1)-v_{1}(0, t+1)\right]<\kappa \\ {\left[0, N_{L}(t)\right]} & \text { otherwise }\end{cases}
$$

where $v_{1}(n, t)$ denotes the value of a type $n=0,1 S$ bank in the first subperiod of period $t$. If all single banks find it optimal to search, then the transition path is given by $N_{S}(t)=N_{L}(t)$ and

$$
N_{L}(t+1)=\sigma+(1-\sigma)\left(1-N_{L}(t)\right) N_{L}(t)
$$

as well as

$$
\Delta v(t)=\Gamma+\kappa+\beta(1-\sigma)\left(1-2 N_{L}(t)+\theta N_{L}(T)\right) \Delta v(t+1)
$$

where $\Delta v(t) \equiv v_{1}(1, t)-v_{1}(0, t)$. Condition (20) implies that this is an equilibrium if and only if $\Delta v(t)>\kappa /\left(N_{L}(t)(1-\sigma) \beta\right)$ for all $t$. 
As $t \rightarrow \infty$ the economy converges to the steady state where all single banks search. However, the convergence is not necessarily monotonic, as shown in Figure $4^{28}$ : When the central bank announces the start of the exit policy, all $S$ banks search if the value of a relationship to a single $S$ bank becomes sufficiently positive, and above $\kappa /\left(N_{L}(t)(1-\sigma) \beta\right)$. When all banks search, many are successful, as all $L$ banks are single. So the number of relationships is maximized at $T+1$ (i.e., $N_{L}$ is minimized) and $N(T+1)$ is relatively small. The value of having a relationship is also maximized: As few $S$ banks will be unmatched at $T$, it becomes less likely for matched $L$ banks to be contacted in the following periods. Similarly, as many $L$ banks are already in a relationship, it is also harder for a single bank to build a new relationship. Following this undershoot, $N_{L}(t)$ creeps back up and gradually converges (with or without oscillations) to its new steady-state value. In the Appendix we derive the transition path for the case when single banks are indifferent between searching and not.

\footnotetext{
${ }^{28}$ In the figure, we set $T=0$.
} 

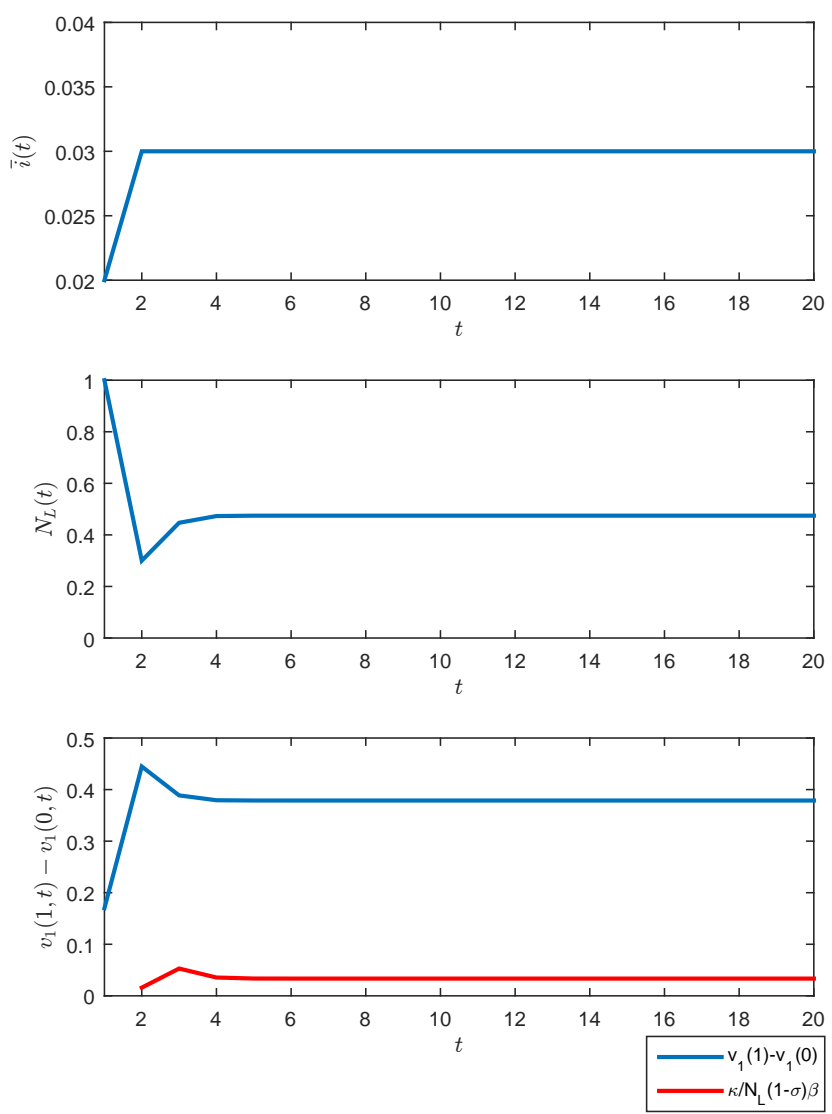

Figure 4: Transition path from floor to symmetric corridor system

\subsection{Regulation on exposure limits}

In April 2014, the Basel Committee on Banking Supervision announced the final standard of the supervisory framework for measuring and controlling large exposures, due to come into force in 2019. According to the BCBS, the large exposure standard includes a general limit applied to all of a bank's exposures to a single counterparty, which is set at $25 \%$ of a bank's Tier 1 capital. This limit also applies to a bank's exposure to identified groups of connected counterparties (i.e., counterparties that are interdependent and likely to fail simultaneously). A tighter limit will apply to 
exposures between banks that have been designated as global systemically important banks (G-SIBs). This limit has been set at $15 \%$ of Tier 1 capital. While there is no counterparty risk in our model, we can still analyze the effect of the regulation on the functioning of the interbank market and its network. To do so, we assume that banks in a relationship can only lend up to a limit $B>0$, equivalent to $x \%$ of their Tier 1 capital.

Since the core market is a centralized market, banks can borrow from many counterparties bearing limited exposure to each of them. Therefore in our simple model, the constraint will not bind in the core market. However, it may bind in the peripheral market. When the exposure limit does not bind, banks trade $R_{S}$ and, as before, bank $S$ does not enter the core market. However, if the exposure limit binds, banks trade $B$ and bank $S$ still holds $R_{S}-B$ if $R_{S}>0$ and $R_{S}+B$ when $R_{S}<0$. In other words, the size of the loan from the $S$ to the $L$ bank is $z\left(R_{S}, R_{L}\right)=B$ if $R_{S}>0$ and $z\left(R_{S}, R_{L}\right)=-B$ otherwise. Then, $S$ banks will enter the core market whenever they still hold too much or too few reserves,

Lemma 2. An $S$ bank enters the core market to borrow $R_{S}+B$ iff $R_{S}+B \leq \hat{R}^{-}$ and enters to lend $R_{S}-B$ iff $R_{S}-B \geq \hat{R}^{+}$, where $\hat{R}^{-}$and $\hat{R}^{+}$are defined in Lemma 1.

Then banks decide on the rate $\hat{i}$ so that bank $L$ takes a share $\theta$ of the total surplus so that the rate for the relationship loan is given by,

$$
\begin{aligned}
1+\hat{i}\left(R_{S}, R_{L} ; B\right) & =(1-\theta) \frac{v_{3}\left(R_{S}, 0,0\right)-v_{3}\left(R_{S}-z\left(R_{S}, R_{L}\right), 0,0\right)}{\beta z\left(R_{S}, R_{L}\right)} \\
& +\theta \frac{V_{3}\left(R_{L}+z\left(R_{S}, R_{L}\right), 0,0\right)-V_{3}\left(R_{L}, 0,0\right)}{\beta z\left(R_{S}, R_{L}\right)}+\frac{c \mathcal{V}}{z\left(R_{S}, R_{L}\right)},
\end{aligned}
$$

and using the expressions for $v_{3}$ and $V_{3}$, and noticing that $i\left(R_{S}\right)=i\left(R_{S}-z\right)$ when 
the exposure limit binds, we obtain

$$
\begin{aligned}
1+\hat{i}\left(R_{S}, R_{L} ; B\right) & =(1-\theta) \frac{\max \left\{\Pi\left(R_{S}\right)-\gamma, 0\right\}-\max \left\{\Pi\left(R_{S}-z\left(R_{S}\right)\right)-\gamma, 0\right\}}{\beta z\left(R_{S}\right)} \\
& +(1-\theta)\left(1+i\left(R_{S}\right)\right)+\theta\left(1+i_{m}(\bar{R})\right)+\frac{c \mathcal{V}}{z\left(R_{S}\right)} .
\end{aligned}
$$

Notice that as $B \rightarrow \infty, z\left(R_{S}\right) \rightarrow R_{S}$ so that $\max \left\{\Pi\left(R_{S}-z\left(R_{S}\right)\right)-\gamma, 0\right\} \rightarrow 0$. Hence, the relationship rate is lower when bank $S$ is lending $\left(z\left(R_{S}\right)>0\right)$ and higher otherwise, as gains from trade are smaller. As a consequence, the value of the relationship is lower and we should expect to have a lower number of relationships in equilibrium. Hence, the regulation on bilateral exposure will distort relationship formation and change the interbank network structure.

\section{Quantitative implications of the OTC interbank market}

Our assumption that banks trade in a centralized market has three important consequences. First any amount of excess reserves drives the interbank rate to the floor $i_{d}$ of the corridor, while any amount of reserves deficit drives the interbank rate to its ceiling $i_{\ell}$. There is only a middle ground when there are no excess reserves (or deficit); then the interbank rate is the midpoint of the corridor. Second, $S$ banks adopt extreme bidding strategies except when the policy rate is in the midpoint of the corridor, in which case they bid zero reserves. Finally, $S$ banks in a relationship never find it optimal to access the core market. These three results are at odds with the data.

We now relax the assumption that the core market is frictionless and instead assume it is over-the-counter (OTC). Below, we summarize the main findings, while detailed analysis and proofs can be found in our companion paper. In this OTC core market, banks can meet pairwise according to a simple matching function, as in Bech and Monnet (2014): a bank ( $S$ or $L$ ) that wants to borrow reserves can only 
meet a bank ( $S$ or $L$ ) with a desire to lend reserves. Then the two banks negotiate over the terms of trade $(x, r)$ where $x$ denotes the loan size and $r$ denotes the loan rate. Although they could, we retain the assumption that banks cannot create a relationship in this market. The rest of the economy is as described above.

Unsurprisingly, we cannot obtain closed-form solutions in the case with an OTC market. However, we show that, as in Bech and Monnet, (1) the OTC rate is now a weighted average of the rates that are bilaterally negotiated, so that it can be strictly within the corridor even though there is an excess or a deficit of reserves. (2) The OTC rate can never lie outside of the corridor, and only the relationship rates can. (3) Banks do not bid an extreme amount of reserves. Finally (4) $S$ banks in a relationship may sometimes find it optimal to enter the core market even though it is costly. Precisely,

Proposition 9. Let the core market be OTC. Consider a pair of banks in a relationship holding balances $R_{S}$ and $R_{L}$ in the periheral market. There are reserves thresholds $\bar{R}^{-}<0$ and $\bar{R}^{+}>0$ such that if $R_{S}+R_{L} \in\left[\bar{R}^{-}, \bar{R}^{+}\right]$then the $S$ bank does not enter the core market. Otherwise the S bank enters the core OTC market. The size of the relationship loan is

$$
z\left(R_{S}, R_{L}\right)= \begin{cases}R_{S} & \text { if } R_{S}+R_{L} \in\left[\bar{R}^{-}, \bar{R}^{+}\right] \\ \left(R_{S}-R_{L}\right) / 2 & \text { otherwise. }\end{cases}
$$

The reason is simple: As the core market is now frictional, sometimes $L$ bank cannot find any other bank to trade or can only find a bank that is not such a good match. This is particularly costly when the $L$ bank is carrying a large reserve surplus or deficit. To insure against such bad luck, the $S$ bank may find it optimal to "share the burden" and pay the fixed entry cost to the core market. This will be so when the $S$ and $L$ banks hold a large reserve surplus or deficit. ${ }^{29}$ Naturally, rates

\footnotetext{
${ }^{29}$ In this case, it may be that the $L$ bank lends to the $S$ bank before the $S$ bank enters the core market.
} 
for relationship trades may still fall outside of the corridor for the same reasons as before, although the formulation for the rate is now more complicated.

In the sequel, we report the following statistics for a parameterized model with an OTC core market: The values of relationship loans, OTC loans, deposits with the central bank by $S$ and $L$ banks, total borrowing at the lending facility by $S$ and $L$ banks, single and matched. We present the details in the companion paper.

\subsection{Parameterization}

The discount factor for banks is $\beta=0.97$. Liquidity shocks $\varepsilon$ for $L$ banks are drawn from a normal distribution $\mathcal{N}(-1,2)$, while liquidity shocks $\xi$ for $S$ banks are drawn from a distribution $\mathcal{N}(1,2)$. Therefore, $S$ banks tend to have liquidity inflows (i.e., potential liquidity providers) and $L$ banks tend to have liquidity outflows (i.e., potential liquidity demanders), even though in aggregate there are no net inflows or outflows. This is consistent with the observations that small banks tend to be liquidity providers to large banks (Stigum and Crescenzi, 2007).

In sub-period 2, the cost for $S$ banks to search and build a new relationship is $\kappa=0.0001$, and the probability of separation is $\sigma=0.1$. In the OTC market, the number of matches is given by

$$
\min \left\{\left(N^{+}\right)^{0.5}\left(N^{-}\right)^{0.5}, N^{+}, N^{-}\right\}
$$

where $N^{+}$is the total measure of banks $\left(S\right.$ or $L$ ) with excess reserves, while $N^{-}$is the total measure of banks with a reserve deficit. The cost for $S$ banks to search in this market is $\gamma=0.0005$. Borrowers and lenders have equal bargaining powers: $\theta=\Theta=0.5$ in both peripheral and core trades. The lending and deposit rates are given by $i_{d}=2 \%$ and $i_{\ell}=4 \%$. In the following, we will examine the effect of applying different policy rates $\bar{i}$. While the parameter values are ad hoc, the implication of the model is qualitatively robust to parameter changes. The numerical algorithm for solving the steady-state equilibrium is described in the Appendix. 
Table 1: Steady-State Equilibrium

[a] Relationship building:

\begin{tabular}{|c|c|c|c|}
\hline & $\eta=1-N_{L}$ & $N_{s}$ & $\mathcal{V}$ \\
\hline \hline $\bar{i}=3.5 \%$ & 0.3438 & 0.0582 & $-7.85 \times 10^{-3}$ \\
\hline $\bar{i}=3.0 \%$ & 0.4062 & 0.0760 & $-8.72 \times 10^{-3}$ \\
\hline $\bar{i}=2.5 \%$ & 0.3438 & 0.0582 & $-7.80 \times 10^{-3}$ \\
\hline
\end{tabular}

[b] Reserve tender:

\begin{tabular}{|c|c|c|c|c|c|}
\hline & $m_{0}$ & $m_{1}$ & $M_{0}$ & $M_{1}$ & Total reserve \\
\hline \hline $\bar{i}=3.5 \%$ & -2.2011 & -1.9772 & -0.1754 & 0.0111 & -2.2354 \\
\hline $\bar{i}=3.0 \%$ & -0.9760 & -1.0016 & 1.0247 & 1.0488 & 0.0482 \\
\hline $\bar{i}=2.5 \%$ & 0.1697 & 0.0775 & 2.1658 & 1.9582 & 2.2325 \\
\hline
\end{tabular}

[c] Relationship loans in perhipheral market:

\begin{tabular}{|c|c|c|c|c|c|}
\hline & avg. $z$ & loans from $S$ banks & avg. $\hat{i}$ & loans with $\hat{i}<i_{d}$ & loans with $\hat{i}>i_{\ell}$ \\
\hline \hline $\bar{i}=3.5 \%$ & 1.1284 & $57.62 \%$ & 1.0350 & $0.88 \%$ & $2.09 \%$ \\
\hline $\bar{i}=3.0 \%$ & 1.1285 & $56.06 \%$ & 1.0300 & $1.85 \%$ & $2.05 \%$ \\
\hline $\bar{i}=2.5 \%$ & 1.1294 & $57.91 \%$ & 1.0249 & $2.02 \%$ & $0.89 \%$ \\
\hline
\end{tabular}

[d] OTC loans in core market:

\begin{tabular}{|c|c|c|c|c|c|}
\hline & avg. $x$ & avg. $r$ & $N^{+}$ & $N^{-}$ & no. of loans \\
\hline \hline $\bar{i}=3.5 \%$ & 1.5268 & 1.0327 & 0.2475 & 0.7152 & 0.2475 \\
\hline $\bar{i}=3.0 \%$ & 1.3986 & 1.0297 & 0.4825 & 0.4739 & 0.4739 \\
\hline $\bar{i}=2.5 \%$ & 1.5267 & 1.0274 & 0.7149 & 0.2481 & 0.2481 \\
\hline
\end{tabular}




\subsection{Effects of policy on rates and volumes}

Table 1 and Figure 5 report equilibrium outcomes under different policy rates. We first consider the equilibrium outcome under a symmetric corridor system with $\bar{i}=$ $0.5\left(i_{d}+i_{\ell}\right)=3 \%$. Banks' reserve demand at the tender is reported in table $1 \mathrm{~b}$ and also plotted in the top left panel of Figure 5. Note that, unlike the benchmark model, this model implies a reserve demand that responds smoothly to changes in the policy rate. As expected, due to their different shock distribution, $S$ banks bid less reserves than $L$ banks. In particular, anticipating a liquidity shock with mean $E(\xi)=1, S$ banks tend to hold a negative reserve position close to -1 , while $L$ banks, anticipating a liquidity shock with mean $E(\varepsilon)=-1$, tend to hold a positive reserve position close to 1 . Since there is no aggregate liquidity shock, the total reserve demand is close to zero (i.e., the minimum reserve requirement) under a symmetric corridor system. The total reserve demand is similar to that in the benchmark model when the policy rate is set at the midpoint of the corridor.

As the policy rate gets closer to the deposit rate, banks find it cheaper to hold more reserve balances to self insure against the risk of accessing the lending facility. As a result, the total demand for reserves increases, and all banks choose a positive initial reserve position. Symmetrically, as the policy rate gets closer to the lending rate, banks prefer to try financing their liquidity needs in the markets, and the total demand for reserves at the tender declines so that most banks choose a negative initial reserve position.

Banks' initial demand for reserves has direct implications for the formation of relationships, the relationship premium and loans, as reported in table 1a and 1c. The number of relationships $\eta=1-N_{L}$ and the value of a relationship, $\mathcal{V}$, are also plotted in the middle two panels of Figure 5.

Under a symmetric corridor, we have seen that $S$ and $L$ banks demand almost opposite amounts of reserves at the tender. So, an $S$ bank and an $L$ bank in a 
relationship are more likely to reach their reserve requirement through relationship trades, and this maximizes the value of a relationship to the $S$ bank (i.e., $|\mathcal{V}|$ is maximized). When the policy rate is closer to the deposit (lending) rate, $S$ and $L$ banks tend to both acquire a positive (negative) initial reserve position, thereby reducing the value of relationships. This explains why both the number of relationships $\eta$ and the number of $S$ banks building relationships $N_{S}$ are maximized under a symmetric corridor. As discussed below, this implies that the interbank network tends to be more tiered under a symmetric corridor than in, e.g., a floor system. As discussed, since $\mathcal{V}$ is negative, an $L$ bank will always be able to borrow at a cheaper rate from its relationship partner, or lend to its partner at a higher rate if it can find a new partner easily.
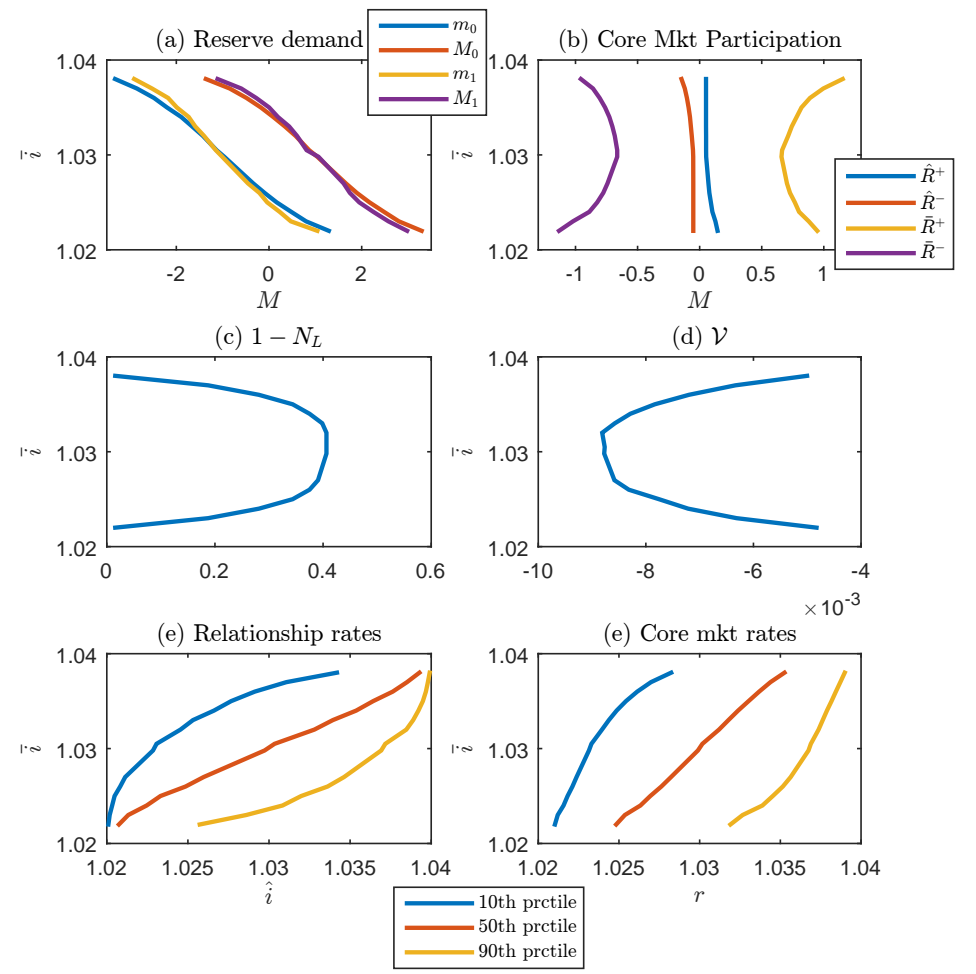

Figure 5: Finding: Policy effects on reserve holding and rates 
Banks' initial reserve demand also affects the functioning of the core market. As shown in table $1 \mathrm{~d}$, given the reserve distribution under a symmetric corridor, the core market is relatively balanced: the number of borrowers $\left(N^{-}\right)$is close to the number of lenders $\left(N^{+}\right)$, and the total number of loans is high. Since finding a partner in the OTC market is easy, more $S$ banks are induced to pay the fixed cost $\gamma$ to trade in the core market. As discussed below, this implies a more complete interbank network. Also, the top right of Figure 5 shows the entry decision in the core market of $S$ banks with and without a relationship. Unlike in the benchmark model, banks in a relationship may also choose to participate in the core market. While single $S$ banks will almost always enter, $S$ banks in a relationship will only enter if their balances (in absolute value), after trading with their $L$ bank, are greater than (approx.) 1. Hence, $S$ banks with a relationship are much less likely to enter the core market, although they might. When the policy rate is close to the midpoint, participation of $S$ banks is maximized. When the policy rate is closer to the deposit (lending) rate, the market becomes unbalanced since banks tend to acquire a positive (negative) initial reserve position. This reduces trades in the core market, the participation of $S$ banks declines and the network becomes less complete. As shown in Figure 5, as the policy rate moves away from the midpoint, the absolute values of the thresholds $\hat{R}^{-}, \hat{R}^{+}, \bar{R}^{-}, \bar{R}^{+}$go up, implying lower participation of $S$ banks.

Finally, let us point out a few interesting observations and the intuition. First, the top left panel of Figure 5 shows the demand for reserves at the tender by $S$ and $L$ banks as a function of their status $n \in\{0,1\}$. Notice that the demand for reserves of banks in a relationship (i.e., $m_{1}, M_{1}$ ) is less interest-elastic than for single banks (i.e., $m_{0}, M_{0}$ ) because a relationship allows risk sharing between two banks that face idiosyncratic liquidity shocks. The reduction in liquidity uncertainty faced by banks in a relationship makes them less responsive to the policy rate.

Second, the bottom panels of Figure 5 show the rates in the peripheral and core markets for different policy rates. Notice that the average relationship rate $E(\hat{i})$ is 
more sensitive to the policy rate than the average core rate $E(r)$. To understand why, note that banks in a relationship have two options to trade liquidity: They can trade at $r$ in the core market and they can trade with the central bank at $i_{d}$ or $i_{\ell}$. When $\bar{i}$ is close to the deposit rate, there is aggregate liquidity surplus, and it becomes more likely that a borrower can borrow at $r$ in the core market, while a lender needs to deposit at the facility at $i_{d}$. Therefore, the relationship rate tends to be between $r$ and $i_{d}$ when the policy rate is below the midpoint of the corridor. Similarly, when $\bar{i}$ is close to the lending rate, it becomes more likely that a lender can lend at $r$ in the core market, while a borrower has to borrow from the facility at $i_{\ell}$. Therefore, the relationship rate tends to be between $r$ and $i_{\ell}$ when the policy rate is above the midpoint of the corridor.

Third, the average loan size in the core market is larger than that in the peripheral market, as shown in Table 1. This is because, as suggested by lemma 1, $S$ banks trade in the core market only when they have large liquidity positions (in absolute terms). Therefore, there are fewer banks with small liquidity needs in the core market. Overall, as the policy market moves away from the midpoint., the number of loans drops but the average loan size goes up in both markets. ${ }^{30}$

Fourth, as Lemma 3 suggests, some relationship loans are traded outside the corridor. Trading below $i_{d}$ (above $i_{\ell}$ ) is more likely to happen when the policy rate is closer to $i_{d}\left(i_{\ell}\right)$, as in that case the spread between $r$ and $i_{d}\left(i_{\ell}\right)$ is small relative to the relationship premium. Figure 6 shows the distributions of rates. Each row shows the frequency of the core rate (left panel) and relationship rate (right panel) for a given policy rate $\bar{i}$. When the policy rate is close to the floor, most trades in the core market are conducted at rates that are also close to the floor. The relationship rate is

\footnotetext{
${ }^{30}$ Interestingly, this is consistent with the US experience under the floor system as reported in Beltran, Bolotnyy and Klee (2015). They point out that "there was a sharp contraction in the number of links in the network during the last quarter of 2008, as fewer banks were transacting with each other" and that "a sizable increase in the average dollar volume transacted per link, which, as we demonstrate later, is largely driven by the exit of many of the smaller banks from the market."
} 
even more skewed toward the floor, and $3 \%$ of the relationship trades are conducted at rates that are below $i_{d} \cdot{ }^{31}$ The situation is reversed when the policy rate is close to $i_{\ell}$, as shown by the lower panel of Figure 6. Therefore, our model can explain why banks trade below the floor (or above $i_{\ell}$ ) and also why we observe apparently unexploited arbitrage opportunities, as reported in Bowman, Gagnon and Leahy (2010). An implication is that policy-makers have no need to worry about interbank trades conducted outside the corridor due to long-term relationships because they are consistent with a well-functioning interbank market. However, one should also be careful in interpreting the interbank market rate as a reference for overnight cost of liquidity, because it may also incorporate a relationship premium, which at times can significantly distort the observed overnight rate.
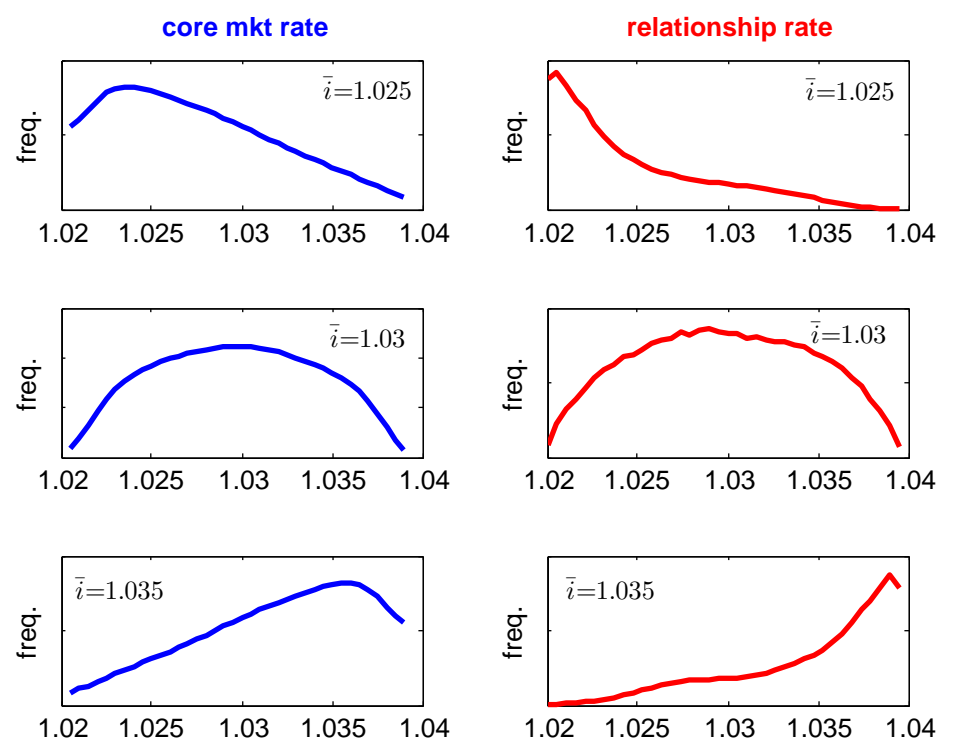

Figure 6: Finding: Policy effects on distributions of relationship and OTC rates

\footnotetext{
${ }^{31}$ Of course, a different parametrization would give us more trades at rates lower than the floor. Also, as $\bar{i}$ gets closer to $i_{d}$, there will be a larger fraction of loans traded below the floor.
} 


\subsection{Network structure}

We now turn to the effect of monetary policy on the network structure. Figure 7 shows the (unweighted) flows of payment for several maintenance periods. When $\gamma=$ 0 , the core market is costless to access. Therefore, whether they are in a relationship or not, $S$ banks will enter. Therefore, after a sufficient number of maintenance periods, all banks will have traded with one another at least once, as shown by the left panel of Figure 7. In this case monetary policy only affects the network structure insofar as it affects the core market tightness.

Now consider the case when accessing the core market is expensive for $S$ banks. We have seen that a relationship is most valuable and the number of relationships is maximized when the policy rate is at the midpoint of the corridor. The network structure is then as shown by the right panel of Figure 7. Since $\gamma$ is large, single $S$ banks choose not to access the core market while $S$ banks in a relationship will also rely on their partners and will not access the core market. So only $L$ banks are active in the core market and provide intermediation for some $S$ banks in the peripheral market.

Finally, suppose the central bank is adopting a more accommodative policy stance and lowers $\bar{i}$ close to $i_{d}$. Then, as $\gamma$ is large, no $S$ bank will access the core market; however, a relationship becomes not so valuable, as all banks are holding enough reserves to satisfy their reserves requirements. Therefore, single $S$ banks have no incentives to build a relationship, and the tiered network structure will slowly vanish as banks are hit by the exogenous separation shock. Then the network structure from the flow of payment will resemble the pattern shown on the middle panel of Figure 7. ${ }^{32,33}$

\footnotetext{
${ }^{32}$ Interestingly, this is consistent with the network change in the fed funds market as reported in Beltran, Bolotnyy and Klee (2015): "many small federal funds lenders began reducing their lending to larger institutions in the core of the network starting in mid-2007."

${ }^{33}$ Again, this model in some way endogenizes bank sizes: even when banks face the same shock distribution, $G=F$, the $L$ banks will appear more active in the interbank market, hence justifying
} 

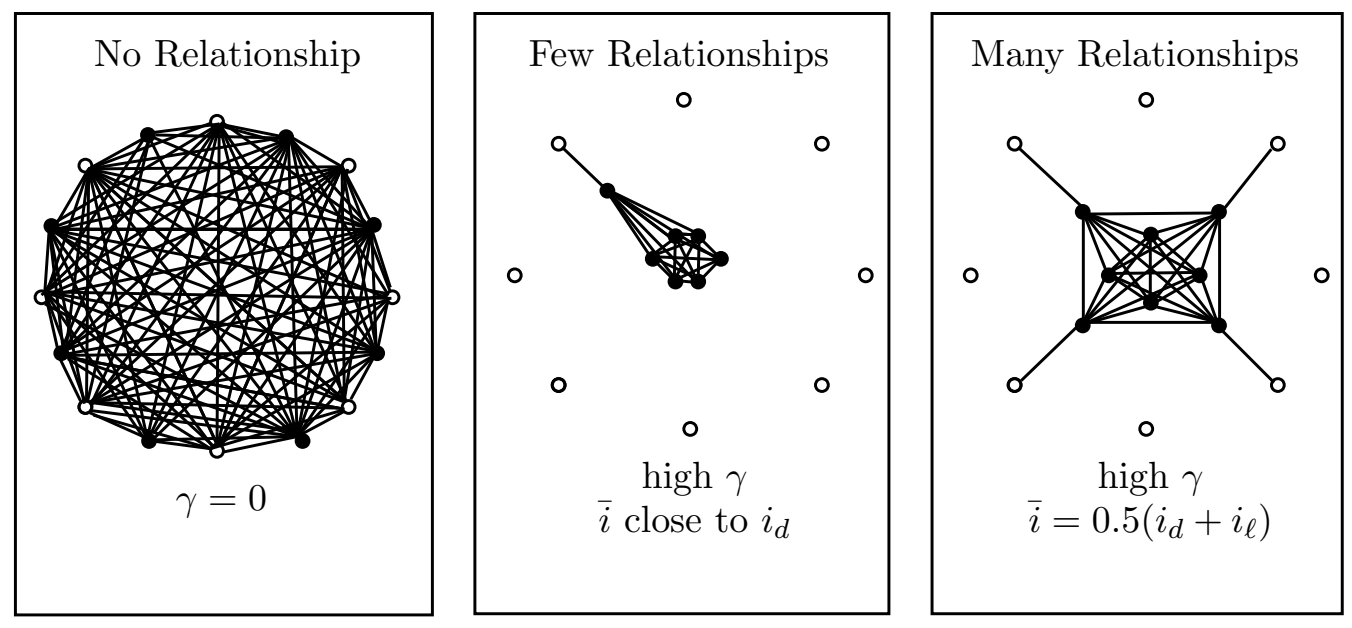

Figure 7: Effect of monetary policy on network structure.

\subsection{Composition of loans}

Figure 8 shows the composition of loans in the interbank market, still as a function of policy rates. As expected, relationship loans vanish when the policy rate is driven to the extremes of the corridor. One interesting aspect of the model is the coexistence of both borrowing and lending at the central bank's standing facilities, which is a property of the data (see Bech and Monnet, 2014). This is inefficient, as some banks have excess reserves (which they deposit with the central bank) while others are short of reserves (which they have to borrow from the central bank). Evidently, the reason for this inefficiency is the friction in the core market. This gives an additional value for building relationship: Banks now have the opportunity to attain their targeted level of reserves by either trading with their preferred bank or by accessing the core market.

the labels " $L$ " and "S." To see that, compare the average trading activities of an $S$ bank and an $L$ bank in a relationship: (i) in the peripheral market, expected trade size is the same for $S$ and $L$ banks in absolute terms; (ii) when both banks enter the OTC core market, the expected trade size is the same for $S$ and $L$ banks because they split their total balance and bring the same amount to the OTC; (iii) when only the $L$ bank enters the OTC market, the $L$ bank has a higher expected trade size. 


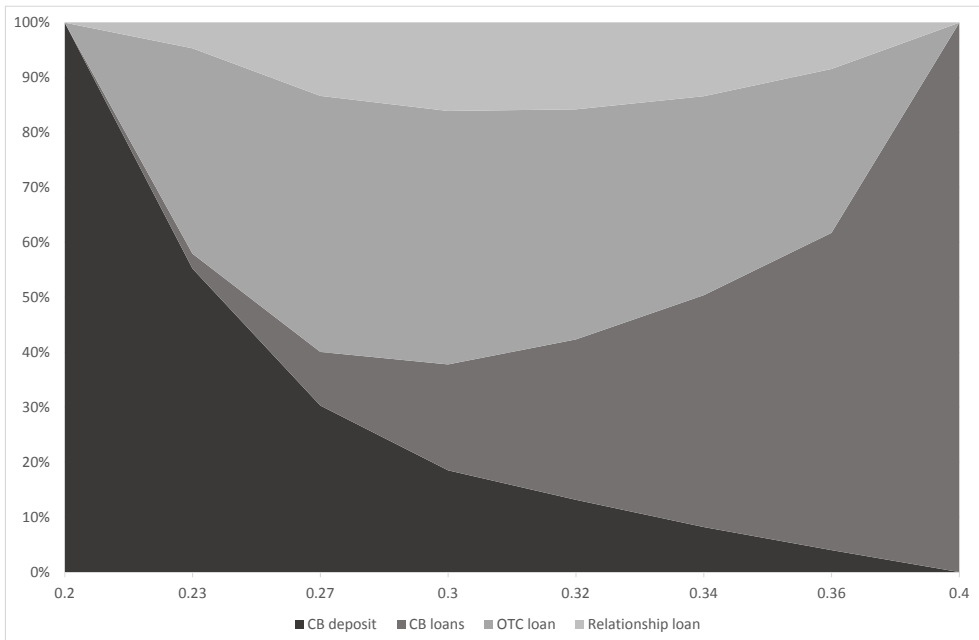

Figure 8: Finding: Policy effects on composition of overnight loans

\subsection{Temporary liquidity expansion/contraction}

Our analysis so far focuses on permanent anticipated changes in monetary policy. We now consider a one-time unanticipated liquidity injection to the banking system. Consider a steady-state equilibrium under a symmetric corridor (i.e., $\bar{i}=3.0 \%$ ). Suppose all banks receive a surprise lump-sum reserve injection $\Delta m \gtrless 0$ from the central bank after the liquidity sale, and receive zero injections in all subsequent periods. This exercise captures a situation in which the central bank conducts a temporary monetary expansion/contraction, with its commitment to restore steadystate policy in the long run.

Crucially, the policy effects of these temporary deviations from a symmetric corridor are different from those of a permanent deviation. Table 2 reports the effects of such intervention on the quantities and prices of interbank loans. Naturally, a liquidity injection drives the core and relationship rates to the floor while a withdrawal drives the rates to $i_{\ell}$. More interestingly, contrary to a permanent liquidity injection, a temporary liquidity injection does not affect the number of relationship 
Table 2: Temporary Liquidity Expansion/Contraction

\begin{tabular}{|c|c|c|c|c||c|c|}
\hline \multirow{2}{*}{$\Delta m$} & \multicolumn{2}{|c||}{ Relationship loans } & \multicolumn{2}{c||}{ OTC loans } \\
\cline { 2 - 7 } & no. of loans & avg. $\hat{i}$ & loans with $\hat{i}<i_{d}$ & loans with $\hat{i}>i_{\ell}$ & no. of loans & avg. $r$ \\
\hline-2 & 0.2031 & 1.0371 & $0.98 \%$ & $3.15 \%$ & 0.1128 & 1.0344 \\
\hline-1 & 0.2031 & 1.0346 & $1.16 \%$ & $2.86 \%$ & 0.2551 & 1.0321 \\
\hline 0 & 0.2031 & 1.0300 & $1.85 \%$ & $2.05 \%$ & 0.4739 & 1.0297 \\
\hline+1 & 0.2031 & 1.0247 & $2.62 \%$ & $1.42 \%$ & 0.2690 & 1.0275 \\
\hline+2 & 0.2031 & 1.0214 & $3.48 \%$ & $1.09 \%$ & 0.1192 & 1.0254 \\
\hline
\end{tabular}

loans. Figure 8 shows that a permanent deviation from a symmetric corridor always reduces the number of loans in the core and peripheral markets. A one-time monetary expansion/contraction also reduces core market loans because the market becomes less balanced, but the effect on relationship loans is quite different. Since banks in a relationship are not subject to search frictions, the number of relationship loans is unaffected. Moreover, since these policies are temporary, the relationship premium is also unaffected. As a consequence, there remains a significant fraction of relationship loans traded below the floor for a liquidity injection and a significant fraction of loans traded above the ceiling for a large liquidity withdrawal. ${ }^{34}$

\subsection{Moving from a floor system to a symmetric corridor}

Finally we compute the transition path when the central bank moves the policy rate from the bottom (i.e., floor system) to the midpoint of the corridor (i.e., symmetric corridor system). The transition paths of $\eta=1-N_{L}$ and $\mathcal{V}$ are plotted in Figure 9. As in the benchmark model, there is an overshoot of relationship formation, as the number of relationships increases immediately in response to the policy change. Afterwards, the number of relationships declines monotonically to its new steady value. As discussed in the benchmark model, $S$ banks' value of long-term relationships also exihibits a non-montonic time path.

\footnotetext{
${ }^{34}$ One can show that, as $\Delta m \rightarrow \infty$, OTC loans vanish but there remains a positive number of relationship loans. Moreover, a positive fraction of relationship loans are traded below the floor.
} 

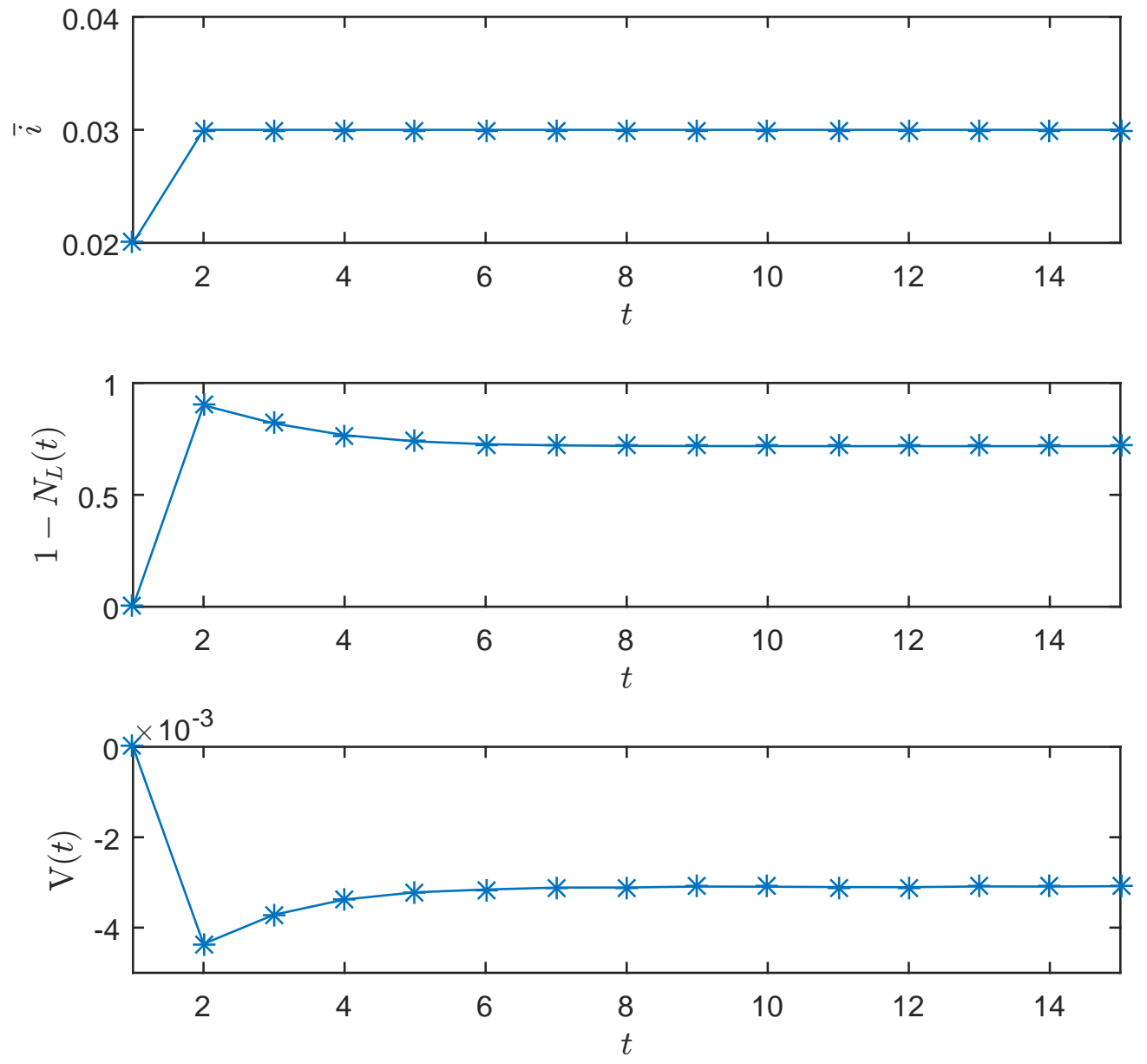

Figure 9: Number of relationships and value of relationship along the transition path

\section{Conclusion}

We presented a model of the unsecured segment of the interbank money market, where banks had incentives to build long-term relationships. The network of interbank loans then arises endogenously and is affected by monetary policy. Therefore, 
the model sheds some light on the policy effects on the interbank network, reserve demand, and interest rate dispersion. We derive testable implications regarding the network structure and the composition, quantities and prices of loans. We show that relationship can help explain some puzzling observations, such as why the floor of the corridor is "soft." The model is simple and tractable and, we believe, captures some important components of the interbank market. As such, it can be a useful tool for policy-makers, e.g., to investigate quantitatively the short-run and long-run effects of running and "exiting" the floor system and the potential effects of various regulatory proposals.

Of course we had to make some simplifying assumptions. For example, we could have assumed that banks in a relationship can meet once more after the core market. However, this would have unnecessarily complicated the analysis of trades in the core market. More importantly, we now assume that $L$ banks can set up a link with only one other bank. It could be interesting to relax this assumption. Also, $S$ banks may have the incentive to create some relationships with each other and delegate access to the core market to one of them, while sharing the access cost. Effectively, they would become large, as they would each bear only a small fraction of the access cost. We have not explored this interesting possibility here, but in the companion paper we study an extension where any pairs of banks can form a relationship and another extension where banks can trade in the OTC market multiple times.

A characteristics of the fed funds market is that small banks are usually cash rich and lend to the large cash-poor banks. As Stigum and Crescenzi (2007) summarize it, "In the fed funds market now, regional banks buy up funds from even tiny banks, use what they need, and resell the remainder in round lots in the New York market. Thus, the fed funds market resembles a river with tributaries: money is collected in many places and then flows through various channels into the New York market. In essence, the nation's smaller banks are the suppliers of fed funds, and the larger bankers are the buyers." Our model obviously encompasses this case and our parametrization 
makes full use of it by imposing two different distributions of the liquidity shocks for the two types of banks: The distribution of shocks for the $S$ banks has a positive mean, while the shock for $L$ banks has a negative mean.

Also, instead of assuming a fixed cost of entry in the core market, we could have assumed that it is reserved for large transactions. Indeed Crescenzi and Stigum (2007) report that "A smaller bank could, of course, shop in the brokers' market and try to pick up an extra, but most don't because the amounts they sell are so small that the cost of trying would outweigh the potential gain. Overnight on $\$ 10$ million is only $\$ 17$, and that's before the phone bill is paid.(...) The brokers' market is really open to only those banks that buy and sell in volume. In fed funds, round lots are $\$ 5$ million, the same as the notional amount on the fed funds futures that trade at the Chicago Board of Trade. A small bank in Iowa that wants to buy $\$ 500,000$ is better off going to its regional correspondent, since the New York brokers are not set up to handle trades of that size." However our fixed-cost assumption is a good assumption for this aspect of the market, and our results would have been essentially the same. Also, notice that, in equilibrium, trade in the core market will be relatively large, and thus consistent with this evidence.

Finally, we have left aside credit risk as well as the juxtaposition of the unsecured and secured segments of the money market. It is clear that credit risk will affect the quality of long-term relationships. Also, we have seen the importance of the secured segment during the recent financial crisis. Adding both elements to the current model would be a natural and very interesting next step. We leave these and other improvements for future research. 


\section{References}

[1] Afonso, G., A. Kovner, and A. Schoar (2012). "The Importance of Trading Relationships in the Fed Funds Market," mimeo, Federal Reserve Bank of New York.

[2] Afonso, G. and R. Lagos (2012). "An Empirical Study of Trade Dynamics in the Fed Funds Market," mimeo, Federal Reserve Bank of New York.

[3] Afonso, G. and R. Lagos (2015). "Trade Dynamics in the Market for Federal Funds." Econometrica 83.1: 263-313.

[4] Allen, F. and A. Babus (2009) "Networks in Finance," in P. Kleindorfer and J. Wind (ed.) Network-based Strategies and Competencies, 367-382.

[5] Allen, F. and G. Douglas (2000) "Financial Contagion." Journal of Political Economy 108, 1-33.

[6] Armantier, O. and A. Copeland (2012). "Assessing the Quality of 'Furfine-based' Algorithms." Staff Report 575, Federal Reserve Bank of New York.

[7] Ashcraft, A. and D. Duffie (2007). "Systemic Illiquidity in the Federal Funds Market." American Economic Review 97(2), 221-225.

[8] Babus, A. (2013) "Endogenous Intermediation in Over-the-Counter Markets," mimeo, Federal Reserve Bank of Chicago.

[9] Bech, M. and E. Atalay (2010). "The topology of the federal funds market." Physica A: Statistical Mechanics and its Applications 389.22: 5223-5246.

[10] Bech, M., C. Bergstrom, R. Garratt, and M. Rosvall (2014). "Mapping Change in the Federal Funds Market." Federal Reserve Bank of New York Staff Reports, no. 507. 
[11] Bech, M. and C. Monnet (2014). "Sorting in the Interbank Market," mimeo, Bank for International Settlements.

[12] Bech, M. and T. Keister (2012). "The Liquidity Coverage Ration and Monetary Policy," mimeo, Bank for International Settlements.

[13] Bech, M. and E. Klee (2011). "The Mechanics of a Graceful Exit: Interest on Reserves and Segmentation in the Federal Funds Market." Journal of Monetary Economics 58, 415-431.

[14] Beltran, D., V. Bolotnyy, and E. Klee (2015). "'Un'-Networking: The Evolution of Networks in the Federal Funds Market," mimeo, Federal Reserve Board of Governors, DC.

[15] Berentsen, A. and C. Monnet (2008). "Monetary Policy in a Channel System." Journal of Monetary Economics 55, 1067-1080.

[16] Berentsen, A., A. Marchesiani, and C. Waller (2011). "Floor Systems for Implementing Monetary Policy: Some Unpleasant Fiscal Arithmetic." Review of Economic Dynamics 17(3), 523-542.

[17] Berentsen, A. and C. Waller (2011). "Price Level Targeting and Stabilization Policy." Journal of Money, Credit, and Banking 43(s2), 559-580.

[18] Blasques, F., F. Brauning, and I. van Lelyveld (2015). "A Dynamic Network Model of The Unsecured Interbank Lending Market." BIS Working Paper No. 491.

[19] Bowman, D., E. Gagnon and M. Leahy (2010). "Interest on Excess Reserves as a Monetary Policy Instrument: The Experience of Foreign Central Banks." Board of Governors of the Federal Reserve System, International Finance Discussion Papers No. 996. 
[20] Brauning, F. and F. Fecht (2012). "Relationship Lending and Peer Monitoring: Evidence from Interbank Payment Data," mimeo, VU University of Amsterdam.

[21] Chang, B. and S. Zhang (2015). "Endogenous Market Making and Network Formation," mimeo, University of Wisconsin-Madison.

[22] Craig, B., and G. Von Peter (2014). "Interbank Tiering and Money Center Banks." Journal of Financial Intermediation 23.3: 322-347.

[23] Duffie, D., N. Gârleanu, and L. Pedersen (2005). "Over the Counter Markets." Econometrica 73.6: 1815-1847.

[24] Elliott, M. L., B. Golub, and M. O. Jackson (2014). "Financial Networks and Contagion." American Economic Review 104.10 (2014): 3115-3153.

[25] European Central Bank (2002) "The Liquidity Management of The ECB." ECB Monthly Bulletin.

[26] European Central Bank (2013) "Euro Money Market Survey," November.

[27] Ennis, H. and J. Weinberg (2013) "Over-The-Counter Loans, Adverse Selection, and Stigma in The Interbank Market." Review of Economic Dynamics 16, 601616.

[28] Furfine, C. (1999) "The Microstructure of the Federal Funds Market." Financial Markets, Institutions \&3 Instruments 8(5), November.

[29] Gofman, M. (2011) "A Network-Based Analysis of Over-the-Counter Markets," mimeo, Wisconsin Business School.

[30] Gofman, M. (2014) "Efficiency and Stability of a Financial Architecture with Too-Interconnected-to-Fail Institutions," mimeo, Wisconsin Business School. 
[31] Hamilton, J. D. (1996) "The Daily Market for Federal Funds." Journal of Political Economy 104, 26-56.

[32] Hartmann, P., M. Manna, and A. Manzanares (2001) "The Microstructure Of The Euro Money Market." Journal of International Money and Finance 20(6), 895-948.

[33] Jackson, M. O. (2010) "Social and Economic Networks." Princeton University Press.

[34] Keister T., A. Martin and J. McAndrews (2008). "Divorcing Money from Monetary Policy." Federal Reserve Bank of New York Economic Policy Review.

[35] Klee, E., and V. Stebunovsy (2013) "Target practice: Monetary policy implementation in a post-crisis environment," manuscript.

[36] Lagos, R., and G. Rocheteau (2009). "Liquidity in asset markets with search frictions." Econometrica 77.2: 403-426.

[37] Lagos, R., G. Rocheteau, and P-O. Weill (2011) "Crises and Liquidity in Overthe-Counter Markets." Journal of Economic Theory 146, 2169-2205.

[38] Lascelles, E. (2009). "Rate Cutting On The Sly." TD Securities Market Musings, May 14.

[39] Leitner, Y. (2005) "Financial Networks: Contagion, Commitment, and Private Sector Bailouts." Journal of Finance 60, 2925-2953.

[40] Li, Y., G. Rocheteau, and P.-O. Weill (2012) "Liquidity and the Threat of Fraudulent Assets." Journal of Political Economy 120, 815-846.

[41] Poole, W. (1968). "Commercial Bank Reserve Management in a Stochastic Model: Implications for Monetary Policy." Journal of Finance 23, 769-791. 
[42] Rajan, R. G. (1992). "Insiders and Outsiders: The Choice Between Informed and Arm's-length Debt." Journal of Finance 47(4):1367-400.

[43] Rocheteau, G. and R. Wright (2013) "Liquidity and Asset Market Dynamics." Journal of Monetary Economics 60, 275-294.

[44] Stigum, M. and A. Crescenzi (2007) Stigum's Money Market, McGraw-Hill Education, 4th Edition.

[45] Vila, A., K. Soramaki, and P. Zimmerman (2010). "The Sterling Unsecured Loan Market During 2006-2008: Insights From Network Theory," manuscript. 


\section{Appendix}

\section{A Proof of Proposition 3}

Proof. Consider a trade between an $S$ bank and an $L$ bank. Recall that

$$
v_{3}(R, L, n)=\max \{\Pi(R)-\gamma, 0\}+\beta\{L+R(1+i(R))\}+\beta \bar{v}_{1}(n) .
$$

If the $S$ bank lends $z$ to the $L$ bank, its payoff is

$$
\begin{aligned}
& v_{3}\left(R_{S}-z, z(1+\hat{i}), 1\right) \\
= & \max \left\{\Pi\left(R_{S}-z\right)-\gamma, 0\right\}+\beta\left\{z(1+\hat{i})+\left(R_{S}-z\right)\left(1+i\left(R_{s}-z\right)\right)\right\}+\beta \bar{v}_{1}(1) .
\end{aligned}
$$

If it does not lend, its payoff depends on whether the $L$ bank has been contacted or not. If $c=1$, then the $L$ bank can credibly threaten to break up the relationship if no trade is conducted in the current period. Therefore, the $S$ bank's payoff without trade is

$$
\begin{aligned}
& v_{3}\left(R_{S}, 0,0\right) \\
= & \max \left\{\Pi\left(R_{S}\right)-\gamma, 0\right\}+\beta\left\{R_{S}\left(1+i\left(R_{S}\right)\right)\right\}+\beta \bar{v}_{1}(0) .
\end{aligned}
$$

In this case, the trade surplus for an $S$ bank is

$$
v_{3}\left(R_{S}-z, z(1+\hat{i}), 1\right)-v_{3}\left(R_{S}, 0,0\right)
$$

In contrast, when $c=0$, the relationship continues even when there is no trade in the current period and the $S$ bank's payoff is

$$
\begin{aligned}
& v_{3}\left(R_{S}, 0,1\right) \\
= & \max \left\{\Pi\left(R_{S}\right)-\gamma, 0\right\}+\beta\left\{R_{S}\left(1+i\left(R_{S}\right)\right)\right\}+\beta \bar{v}_{1}(1) .
\end{aligned}
$$

In that case, the trade surplus for an $S$ bank is

$$
v_{3}\left(R_{S}-z, z(1+\hat{i}), 1\right)-v_{3}\left(R_{S}, 0,1\right)
$$


As a result, the $S$ bank's trade surplus conditional on $c$ is

$$
\begin{aligned}
& T S_{S}(z, i ; c) \\
= & v_{3}\left(R_{S}-z, 0,0\right)-v_{3}\left(R_{S}, 0,0\right)+\beta z(1+\hat{i})+c \beta\left[\bar{v}_{1}(1)-\bar{v}_{1}(0)\right] \\
= & \max \left\{\Pi\left(R_{S}-z\right)-\gamma, 0\right\}-\max \left\{\Pi\left(R_{S}\right)-\gamma, 0\right\} \\
& +\beta\left\{z(1+\hat{i})+\left(R_{S}-z\right)\left(1+i\left(R_{S}-z\right)\right)-R_{S}\left(1+i\left(R_{S}\right)\right)\right\}+c \beta(1-\sigma)\left[v_{1}(1)-v_{1}(0)\right] .
\end{aligned}
$$

Since an $S$ bank cannot credibly end a relationship, the trade surplus for an $L$ bank with reserves $R_{L}$ is always

$$
V_{3}\left(R_{L}+z, z(1+\hat{i}), 1\right)-V_{3}\left(R_{L}, 0,1\right)
$$

where

$$
\begin{aligned}
V_{3}\left(R_{L}+z,-z(1+\hat{i}), 1\right) & =\beta\left(R_{L}+z\right)\left(1+i_{m}\right)-\beta z(1+\hat{i})+\beta \bar{V}_{1}(1) \\
V_{3}\left(R_{L}, 0,1\right) & =\beta R_{L}\left(1+i_{m}\right)+\beta \bar{V}_{1}(1) .
\end{aligned}
$$

Hence, its surplus conditional on $c$ is

$$
\begin{aligned}
& T S_{L}(z, \hat{i} ; c) \\
= & V_{3}\left(R_{L}+z, z(1+\hat{i}), 1\right)-V_{3}\left(R_{L}, 0, c\right) \\
= & \beta z\left(1+i_{m}\right)-\beta z(1+\hat{i}) .
\end{aligned}
$$

Then, the total surplus from a loan of size $z$ is

$$
\begin{aligned}
T S(z ; c)= & v_{3}\left(R_{S}-z, 0,0\right)-v_{3}\left(R_{S}, 0,0\right)+V_{3}\left(R_{L}+z, 0,0\right)-V_{3}\left(R_{L}, 0,0\right) \\
& +c \beta(1-\sigma)\left[v_{1}(1)-\beta v_{1}(0)\right] \\
= & \max \left\{\Pi\left(R_{S}-z\right)-\gamma, 0\right\}-\max \left\{\Pi\left(R_{S}\right)-\gamma, 0\right\} \\
& +\beta\left\{\left(R_{S}-z\right)\left(1+i\left(R_{S}-z\right)\right)-R_{S}\left(1+i\left(R_{S}\right)\right)\right\}+c \beta(1-\sigma)\left[v_{1}(1)-v_{1}(0)\right] \\
& +\beta z\left(1+i_{m}\right)
\end{aligned}
$$


Since $\gamma>0$ and $i_{m} \in\left[i_{d}, i_{\ell}\right]$, it is straightforward to show that $R_{s}=\arg \max _{z} T S(z)$ and hence the total surplus from the trade is

$T S\left(R_{S} ; c\right)=-\max \left\{\Pi\left(R_{S}\right)-\gamma, 0\right\}-\beta R_{S}\left(1+i\left(R_{S}\right)\right)+c \beta(1-\sigma)\left[v_{1}(1)-v_{1}(0)\right]+\beta R_{S}\left(1+i_{m}\right)$.

Setting $T S_{L}=(1-\theta) T S\left(R_{S}\right)$, we obtain

$$
\begin{aligned}
1+\hat{i}\left(R_{S}, R_{L}, c\right)= & (1-\theta) \frac{\max \left\{\beta\left[1+i\left(R_{S}\right)\right] R_{S},-\gamma+\beta\left[1+i_{m}(\bar{R})\right] R_{S}\right\}}{\beta R_{S}} \\
& +\theta\left[1+i_{m}(\bar{R})\right]+(1-\theta) \frac{c \mathcal{V}}{R_{S}} .
\end{aligned}
$$

Therefore, the $S$ bank will always lend $z$ to the $L$ bank and choose not to participate in the core market. 


\section{B Value in sub-period 2}

The expected value of a matched $L$ bank with reserve holdings $M$ before the shock is

$$
\begin{aligned}
& V_{2}(M, 1 ; \mathbf{M}) \\
= & \int_{-\infty}^{\infty} \int_{-\infty}^{\infty} E_{c}\left[V_{3}\left(M+\varepsilon+m_{1}+\xi,-\left[1+\hat{i}\left(m_{1}+\xi, c\right)\right]\left(m_{1}+\xi\right) ; 0\right)\right] d G(\varepsilon) d F(\xi) \\
& +\beta\left[\bar{V}_{1}(1)-\bar{V}_{1}(0)\right] . \\
= & \int_{-\infty}^{\infty} \int_{-\infty}^{\infty} E_{c}\left[\beta(M+\varepsilon)\left(1+i_{m}(\bar{R})\right)-\beta\left[\hat{i}\left(m_{1}+\xi, c\right)-i_{m}(\bar{R})\right]\left(m_{1}+\xi\right)\right] d G(\varepsilon) d F(\xi)+\beta \bar{V}_{1}(1) .
\end{aligned}
$$

so that

$$
\frac{\partial V_{2}(M, 1 ; \mathbf{M})}{\partial M}=\beta\left(1+i_{m}(\bar{R})\right)
$$

Similarly, the expected value of a matched $S$ bank with reserve holdings $m$ before the shock is

$$
\begin{aligned}
& v_{2}\left(m_{1}, 1 ; \mathbf{M}\right) \\
= & \int_{-\infty}^{\infty} E_{c}\left[v_{3}\left(0,\left[1+\hat{i}\left(m_{1}+\xi, c\right)\right]\left(m_{1}+\xi\right) ; 0\right)\right] d F(\xi)+\beta\left[\bar{v}_{1}(1)-\bar{v}_{1}(0)\right] . \\
= & \int_{-\infty}^{\infty} E_{c} \beta\left[1+\hat{i}\left(m_{1}+\xi, c\right)\right]\left(m_{1}+\xi\right) d F(\xi)+\beta \bar{v}_{1}(1) . \\
= & \int_{-\infty}^{\infty} \beta\left[(1-\theta) \max \left\{\Pi\left(m_{1}+\xi\right)-\gamma, 0\right\}+\beta\left\{\left(m_{1}+\xi\right)\left(1+i\left(m_{1}+\xi\right)\right)\right\}\right. \\
& \left.+\theta\left(1+i_{m}(\bar{R})\right)\left(m_{1}+\xi\right)+\beta E_{c}[c \mathcal{V}]\right] d F(\xi)+\beta \bar{v}_{1}(1) . \\
= & \int_{-\infty}^{\infty} \beta\left(1+i_{m}(\bar{R})\right)\left(m_{1}+\xi\right)-\beta(1-\theta)\left[\mathbb{I}_{e} \frac{\gamma}{\beta}+\mathbb{I}_{n e}\left[i_{m}(\bar{R})-i\left(m_{1}+\xi\right)\right]\left(m_{1}+\xi\right)\right] d F(\xi) \\
& +\beta E_{c}[c \mathcal{V}]+\beta \bar{v}_{1}(1) .
\end{aligned}
$$

so that, using $\mathbb{I}_{e}=1$ when the bank decides to enter the core market and zero otherwise and $\mathbb{I}_{n e}$ is 1 when the bank opts to stay out of the core market and zero if 
it chooses to enter,

$$
\begin{aligned}
\frac{\partial v_{2}\left(m_{1}, 1 ; \mathbf{M}\right)}{\partial m_{1}}= & \beta\left(1+i_{m}(\bar{R})\right)+\beta(1-\theta) \int_{-\infty}^{\infty} \mathbb{I}_{n e}\left[i\left(m_{1}+\xi\right)-i_{m}(\bar{R})\right] d F(\xi) \\
= & \beta\left(1+i_{m}(\bar{R})\right)+\beta(1-\theta) \int_{\frac{-\gamma}{\beta\left(i_{\ell}-i_{m}(\bar{R})\right)}-m_{1}}^{\frac{\gamma}{\beta\left(i_{m}(\bar{R})-i_{d}\right)}-m_{1}}\left[i\left(m_{1}+\xi\right)-i_{m}(\bar{R})\right] d F(\xi) \\
= & \beta\left(1+i_{m}(\bar{R})\right)+\beta(1-\theta) \\
& \left.\left\{\int_{-m_{1}}^{\frac{\gamma}{\beta\left(i_{m}(\bar{R})-i_{d}\right)}-m_{1}}\left[i_{d}-i_{m}(\bar{R})\right] d F(\xi)+\int_{\frac{-\gamma}{\beta\left(i_{\ell}-i_{m}(\bar{R})\right)}-m_{1}}^{-m_{1}}-i_{m}(\bar{R})\right] d F(\xi)\right\} \\
= & \beta\left(1+i_{m}(\bar{R})\right)+\beta(1-\theta)\left[i_{d}-i_{m}(\bar{R})\right]\left[F\left(\frac{\gamma}{\beta\left(i_{m}(\bar{R})-i_{d}\right)}-m_{1}\right)-F\left(-m_{1}\right)\right] \\
& +\beta(1-\theta)\left[i_{\ell}-i_{m}(\bar{R})\right]\left[F\left(-m_{1}\right)-F\left(\frac{-\gamma}{\beta\left(i_{\ell}-i_{m}(\bar{R})\right)}-m_{1}\right)\right]
\end{aligned}
$$

Notice that if $i_{m}(\bar{R})=\left(i_{\ell}+i_{d}\right) / 2$ then $\frac{\partial v_{2}\left(m_{1}, 1 ; \mathbf{M}\right)}{\partial m_{1}}=\beta\left(1+i_{m}(\bar{R})\right)$.

Using (7) the expected value of a single $L$ bank holding $M$ units of reserves before the shock is

$$
V_{2}(M, 0 ; \mathbf{M})=\int_{-\infty}^{\infty} \beta\left[(M+\varepsilon)\left(1+i_{m}(\bar{R})\right)\right] d G(\varepsilon)+\beta\left(1-N_{S}\right) \bar{V}_{1}(0)+\beta N_{S} \bar{V}_{1}(1)
$$

so that

$$
\frac{\partial V_{2}(M, 0 ; \mathbf{M})}{\partial M}=\beta\left(1+i_{m}(\bar{R})\right)
$$

Using (8) we can write:

$$
\begin{aligned}
v_{2}\left(m_{0}, 0 ; \mathbf{M}\right) & =\int_{-\infty}^{\infty} \max \{\Pi(m+\xi)-\gamma, 0\}+\beta\{(m+\xi)(1+i(m+\xi))\} d F(\xi)+v(0) \\
& =\int_{-\infty}^{\infty} \mathbb{I}_{e}\left[\beta\left(m_{0}+\xi\right)\left(1+i_{m}(\bar{R})\right)-\gamma\right]+\beta \mathbb{I}_{n e}\left(m_{0}+\xi\right)\left[1+i\left(m_{0}+\xi\right)\right] d F(\xi)+v(0)
\end{aligned}
$$

where $v(0)=\max \left\{\beta v_{1}(0),-\kappa+N_{L} \beta \bar{v}_{1}(1)+\left(1-N_{L}\right) \beta v_{1}(0)\right\}$ determines the value 
of searching when single. So

$$
\begin{aligned}
& \frac{\partial v_{2}\left(m_{0}, 0 ; \mathbf{M}\right)}{\partial m_{0}}=\int_{-\infty}^{\infty} \mathbb{I}_{e} \beta\left(1+i_{m}(\bar{R})\right)+\mathbb{I}_{n e} \beta\left(1+i\left(m_{0}+\xi\right)\right) d F(\xi) \\
& =\beta\left(1+i_{m}(\bar{R})\right)+\int_{-\infty}^{\infty} \mathbb{I}_{n e} \beta\left(i\left(m_{0}+\xi\right)-i_{m}(\bar{R})\right) d F(\xi) \\
& =\beta\left(1+i_{m}(\bar{R})\right)+\int_{\frac{-\gamma}{\beta\left(i_{\ell}-i_{m}(\bar{R})\right)}-m_{0}}^{\frac{\gamma}{\beta\left(i_{m}(\bar{R})-i_{d}\right)}-m_{0}} \beta\left(i\left(m_{0}+\xi\right)-i_{m}(\bar{R})\right) d F(\xi) \\
& =\beta\left(1+i_{m}(\bar{R})\right)+\beta\left[i_{d}-i_{m}(\bar{R})\right]\left[F\left(\frac{\gamma}{\beta\left(i_{m}(\bar{R})-i_{d}\right)}-m_{0}\right)-F\left(-m_{0}\right)\right] \\
& +\beta\left[i_{\ell}-i_{m}(\bar{R})\right]\left[F\left(-m_{0}\right)-F\left(\frac{-\gamma}{\beta\left(i_{\ell}-i_{m}(\bar{R})\right)}-m_{0}\right)\right] \text {. }
\end{aligned}
$$




\section{Proof of Proposition 6}

Proof. Recall that the search strategy of single $S$ banks is given by (13). Using (12), we have that if $N_{S}=N_{L}$ (i.e., all single $S$ banks search), then $N_{L}$ solves

$$
(1-\sigma) N_{L}^{2}+\sigma N_{L}-\sigma=0
$$

or

$$
N_{L}^{*}=\frac{-\sigma+\sqrt{\sigma^{2}+4(1-\sigma) \sigma}}{2(1-\sigma)},
$$

and if $N_{S}=0$ (i.e., no $S$ banks search) then $N_{L}=1$. That is, if none of the single $S$ banks search, then all $L$ banks are single. Let

$$
\Gamma \equiv \int_{-\infty}^{\infty}\left\{\beta \theta\left[\mathbb{I}_{e} \frac{\gamma}{\beta}+\mathbb{I}_{n e}\left[\frac{i_{\ell}+i_{d}}{2}-i(\xi)\right] \xi\right]\right\} d F(\xi)
$$

be the expected per-period benefit of a relationship for the $S$ bank when the interbank market is in the midpoint of the corridor. Then

Lemma 10. If $\bar{i} \neq\left(i_{\ell}+i_{d}\right) / 2$ then $v_{1}(1)=v_{1}(0)$ so that $N_{S}=0$ and there is no relationship. When $\bar{i}=\left(i_{\ell}+i_{d}\right) / 2$ then either $N_{S}=N_{L}^{*}$ and

$$
v_{1}(1)-v_{1}(0) \geq \frac{2}{\beta\left[-\sigma+\sqrt{\sigma^{2}+4(1-\sigma) \sigma}\right]} \kappa
$$

or $N_{S}=\bar{N}_{S}$ and

$$
v_{1}(1)-v_{1}(0)=\frac{\left[(1-\sigma) \bar{N}_{S}+\sigma\right]}{\sigma(1-\sigma) \beta} \kappa
$$

where $\bar{N}_{S}$ is implicitly given by

$$
\frac{\sigma \beta(1-\sigma)}{\left[(1-\sigma) \bar{N}_{S}+\sigma\right]} \frac{\Gamma}{\left[1-\beta(1-\sigma)\left[1-\bar{N}_{S}(1-\theta)\right]\right]}=\kappa,
$$

or $N_{S}=0$ and $N_{L}=1$, and

$$
(1-\sigma) \beta\left[v_{1}(1)-v_{1}(0)\right]<\kappa
$$


When $\bar{i} \neq\left(i_{\ell}+i_{d}\right) / 2$ then $v_{1}(1)=v_{1}(0)$. (13) implies that $N_{S}=0$ and there is no relationship. When $\bar{i}=\left(i_{\ell}+i_{d}\right) / 2$ then proposition (4) implies that $m_{0}=m_{1}=0$ and $v_{1}(n)=v_{2}(0, n ; \mathbf{0})$. Suppose first $N_{S}$ is interior. Since $i_{m}(\bar{R})=\bar{i}$ we have

$$
\begin{aligned}
v_{2}(0,0 ; \mathbf{M}) & =\int_{-\infty}^{\infty} \mathbb{I}_{e}\left[\beta \xi\left(1+\frac{i_{\ell}+i_{d}}{2}\right)-\gamma\right]+\beta \mathbb{I}_{n e}[1+i(\xi)] \xi d F(\xi)+v(0) \\
& =\int_{-\infty}^{\infty} \beta\left(1+\frac{i_{\ell}+i_{d}}{2}\right) \xi-\beta\left[\mathbb{I}_{e} \frac{\gamma}{\beta}+\mathbb{I}_{n e}\left[\frac{i_{\ell}+i_{d}}{2}-i(\xi)\right] \xi\right] d F(\xi)+v(0)
\end{aligned}
$$

where $v(0)=\max \left\{\beta v_{1}(0),-\kappa+N_{L} \beta \bar{v}_{1}(1)+\left(1-N_{L}\right) \beta v_{1}(0)\right\}$ determines the value of searching when single. Also

$$
\begin{aligned}
v_{2}(0,1 ; \mathbf{M}) & =\int_{-\infty}^{\infty} \beta\left(1+\frac{i_{\ell}+i_{d}}{2}\right) \xi-\beta(1-\theta)\left[\mathbb{I}_{e} \frac{\gamma}{\beta}+\mathbb{I}_{n e}\left[\frac{i_{\ell}+i_{d}}{2}-i(\xi)\right] \xi\right] d F(\xi) \\
& +\beta E_{c}[c \mathcal{V}]+\beta \bar{v}_{1}(1) .
\end{aligned}
$$

Therefore

$$
\begin{aligned}
v_{1}(1)-v_{1}(0)= & v_{2}(0,1, \mathbf{0})-v_{2}(0,0,0) \\
= & \Gamma+\beta E_{c}[c(1-\theta) \mathcal{V}]+\beta \bar{v}_{1}(1)-v(0) \\
= & \Gamma-\beta E_{c}\left[c(1-\theta)(1-\sigma)\left[v_{1}(1)-v_{1}(0)\right]\right] \\
& +\beta \bar{v}_{1}(1)-\max \left\{\beta v_{1}(0),-\kappa+N_{L} \beta \bar{v}_{1}(1)+\left(1-N_{L}\right) \beta v_{1}(0)\right\}
\end{aligned}
$$

and using $\bar{v}_{1}(1)=(1-\sigma) v_{1}(1)+\sigma v_{1}(0)$ we obtain,

$$
\begin{aligned}
v_{1}(1)-v_{1}(0)= & \Gamma-\beta E_{c}\left[c(1-\theta)(1-\sigma)\left[v_{1}(1)-v_{1}(0)\right]\right] \\
& +\min \left\{\beta(1-\sigma)\left[v_{1}(1)-v_{1}(0)\right], \kappa+\left(1-N_{L}\right) \beta(1-\sigma)\left[v_{1}(1)-v_{1}(0)\right]\right\} \\
= & \Gamma-\beta E_{c}\left[c(1-\theta)(1-\sigma)\left[v_{1}(1)-v_{1}(0)\right]\right]+\beta(1-\sigma)\left[v_{1}(1)-v_{1}(0)\right]
\end{aligned}
$$

where the last equality follows from the fact that the solution for $N_{S}$ is interior. In this case, $E_{c}[c]=N_{S}$ and

$$
v_{1}(1)-v_{1}(0)=\frac{\Gamma}{1-\beta(1-\sigma)\left[1-N_{S}(1-\theta)\right]} .
$$


Hence, to find $\bar{N}_{S}$ we need to solve

$$
N_{L} \beta(1-\sigma)\left[v_{1}(1)-v_{1}(0)\right]=\kappa .
$$

Using (12) evaluated at $\bar{N}_{S}$, we obtain

$$
\frac{\sigma(1-\sigma) \beta}{\left[(1-\sigma) \bar{N}_{S}+\sigma\right]}\left[v_{1}(1)-v_{1}(0)\right]=\kappa,
$$

and using $v_{1}(1)-v_{1}(0)$ we obtain

$$
\frac{\sigma \beta(1-\sigma)}{\left[(1-\sigma) \bar{N}_{S}+\sigma\right]} \frac{\Gamma}{\left[1-\beta(1-\sigma)\left[1-\bar{N}_{S}(1-\theta)\right]\right]}=\kappa,
$$

which implicitly defines $\bar{N}_{S}$. Also, since

$$
N_{L}=\frac{\sigma}{(1-\sigma) N_{S}+\sigma}
$$

we get

$$
\frac{\bar{N}_{L} \beta(1-\sigma)}{\left[1-\beta(1-\sigma)\left[1-\bar{N}_{S}(1-\theta)\right]\right]} \Gamma=\kappa,
$$

When all single $S$ banks prefer to search and the solution $N_{S}$ is not interior, then

$$
\begin{aligned}
v_{1}(1)-v_{1}(0)= & \Gamma-\beta E_{c}\left[c(1-\theta)(1-\sigma)\left[v_{1}(1)-v_{1}(0)\right]\right] \\
& +\kappa+\left(1-N_{L}\right) \beta(1-\sigma)\left[v_{1}(1)-v_{1}(0)\right] \\
= & \Gamma-\beta N_{S}(1-\theta)(1-\sigma)\left[v_{1}(1)-v_{1}(0)\right] \\
& +\kappa+\left(1-N_{L}\right) \beta(1-\sigma)\left[v_{1}(1)-v_{1}(0)\right] \\
= & \frac{\Gamma+\kappa}{1-\beta(1-\sigma)\left(1-N_{L}-N_{S}(1-\theta)\right)} .
\end{aligned}
$$

This equilibrium exists if and only if

$$
N_{L}^{*}(1-\sigma) \beta \frac{\Gamma+\kappa}{1-\beta(1-\sigma)\left(1-N_{L}^{*}-N_{S}^{*}(1-\theta)\right)}>\kappa
$$

or

$$
\frac{N_{L}^{*}(1-\sigma) \beta}{\left[1-\beta(1-\sigma)\left(1-N_{S}^{*}(1-\theta)\right)\right]} \Gamma>\kappa .
$$


Finally, if no single banks search (i.e., $N_{S}=0$ ), then

$$
v_{1}(1)-v_{1}(0)=\frac{\Gamma}{1-\beta(1-\sigma)}
$$

This equilibrium exists if and only if

$$
\frac{(1-\sigma) \beta}{1-\beta(1-\sigma)} \Gamma<\kappa .
$$

It is clear that the parameter space defines three distinct and non-overlapping but connected regions. In each of these regions the equilibrium is well defined and unique. So the equilibrium is unique. 


\section{Proof of Lemma 7}

Proof. Relationship benefits

$$
\begin{aligned}
\Gamma & \equiv \int_{-\infty}^{\infty}\left\{\beta \theta\left[\mathbb{I}_{e} \frac{\gamma}{\beta}+\mathbb{I}_{n e}\left[\frac{i_{\ell}+i_{d}}{2}-i(\xi)\right] \xi\right]\right\} d F(\xi) \\
& =\beta \theta\left[\int_{\hat{R}^{+}}^{\infty} \frac{\gamma}{\beta}+\int_{-\infty}^{\hat{R}^{-}} \frac{\gamma}{\beta}+\int_{\hat{R}^{-}}^{\hat{R}^{+}}\left[\frac{i_{\ell}+i_{d}}{2}-i(\xi)\right] \xi\right] d F(\xi) \\
& =\beta \theta\left[\frac{\gamma}{\beta}\left[1-F\left(\hat{R}^{+}\right)\right]+\frac{\gamma}{\beta} F\left(\hat{R}^{-}\right)+\int_{0}^{\hat{R}^{+}}\left[\frac{i_{\ell}+i_{d}}{2}-i_{d}\right] \xi+\int_{\hat{R}^{-}}^{0}\left[\frac{i_{\ell}+i_{d}}{2}-i_{\ell}\right] \xi\right] d F(\xi) \\
& =\beta \theta\left[\frac{\gamma}{\beta}\left[1-F\left(\hat{R}^{+}\right)\right]+\frac{\gamma}{\beta} F\left(\hat{R}^{-}\right)+\left[\frac{i_{\ell}-i_{d}}{2}\right]\left(\int_{0}^{0} \xi-\int_{\hat{R}^{-}}^{0} \xi\right) d F(\xi)\right]
\end{aligned}
$$

When the variance of the shock $\xi$ increases, $S$ banks will find it profitable to enter more often. Therefore, more weight is placed on $\gamma / \beta$ relative to the other component of $\Gamma$, which is smaller than $\gamma / \beta$. Hence, $\Gamma$ increases in the variance of the shock $\xi$. Also,

$$
\begin{aligned}
\frac{1}{\beta \theta} d \Gamma & =-\frac{\gamma}{\beta} f\left(R^{+}\right) d R^{+}+\left[\frac{i_{\ell}+i_{d}}{2}-i_{d}\right] R^{+} f\left(R^{+}\right) d R^{+} \\
& =-\frac{\gamma}{\beta} f\left(R^{+}\right) d R^{+}+\frac{\gamma}{\beta}\left[\frac{i_{\ell}-i_{d}}{2}\right] \frac{1}{\left(i_{m}(\bar{R})-i_{d}\right)} f\left(R^{+}\right) d R^{+} \\
& =-\frac{\gamma}{\beta}\left[1-\left[\frac{i_{\ell}-i_{d}}{2}\right] \frac{1}{\left(i_{m}(\bar{R})-i_{d}\right)}\right] f\left(R^{+}\right) d R^{+}
\end{aligned}
$$

and

$$
\begin{aligned}
\frac{1}{\beta \theta} d \Gamma & =\frac{\gamma}{\beta} f\left(R^{-}\right) d R^{-}-\left[\frac{i_{\ell}+i_{d}}{2}-i_{\ell}\right] R^{-} f\left(R^{-}\right) d R^{-} \\
& =\frac{\gamma}{\beta} f\left(R^{-}\right) d R^{-}-\frac{\gamma}{\beta}\left[\frac{i_{\ell}-i_{d}}{2}\right] \frac{1}{\left(i_{\ell}-i_{m}(\bar{R})\right)} f\left(R^{-}\right) d R^{-} \\
& =\frac{\gamma}{\beta}\left[1-\left[\frac{i_{\ell}-i_{d}}{2}\right] \frac{1}{\left(i_{\ell}-i_{m}(\bar{R})\right)}\right] f\left(R^{-}\right) d R^{-}
\end{aligned}
$$


Hence, assuming that $f(x)$ converges fast enough to zero as $x \rightarrow \infty$ or $-\infty$,

$$
\frac{\partial \Gamma}{\partial R^{+}}= \begin{cases}0 & \text { if } \bar{R} \geq 0 \\ -\beta \theta \frac{\gamma}{2 \beta} f\left(R^{+}\right) d R^{+} & \text {if } \bar{R}<0\end{cases}
$$

while

$$
\frac{\partial \Gamma}{\partial R^{-}}=\left\{\begin{array}{ll}
0 & \text { if } \bar{R} \leq 0 \\
\beta \theta \frac{\gamma}{2 \beta} f\left(R^{-}\right) d R^{-} & \text {if } \bar{R}>0
\end{array} .\right.
$$

Therefore, if $i_{\ell}-i_{d}$ increases, then

$$
\begin{aligned}
\left.\frac{\partial \Gamma}{\partial\left(i_{\ell}-i_{d}\right)}\right|_{\bar{i}=\frac{i_{\ell}+i_{d}}{2}} & =\beta \theta\left(i_{\ell}-i_{d}\right)\left(\int_{0}^{\hat{R}^{+}} \xi-\int_{\hat{R}^{-}}^{0} \xi\right) d F(\xi)>0 \\
\left.\frac{\partial \Gamma}{\partial\left(i_{\ell}-i_{d}\right)}\right|_{\bar{i}=i_{\ell}} & =-\beta \theta \frac{\gamma}{2 \beta} f\left(R^{+}\right) \frac{\partial R^{+}}{\partial\left(i_{\ell}-i_{d}\right)}+\beta \theta\left(i_{\ell}-i_{d}\right)\left(\int_{0}^{\hat{R}^{+}} \xi-\int_{\hat{R}^{-}}^{0} \xi\right) d F(\xi)>0 \\
\left.\frac{\partial \Gamma}{\partial\left(i_{\ell}-i_{d}\right)}\right|_{\bar{i}=i_{d}} & =\beta \theta \frac{\gamma}{2 \beta} f\left(R^{-}\right) \frac{\partial R^{-}}{\partial\left(i_{\ell}-i_{d}\right)}+\beta \theta\left(i_{\ell}-i_{d}\right)\left(\int_{0}^{\hat{R}^{+}} \xi-\int_{\hat{R}^{-}}^{0} \xi\right) d F(\xi)>0 .
\end{aligned}
$$

Hence $\Gamma$ (measuring the gains from relationships) increases as the corridor size increases. Also

$$
\begin{aligned}
\left.\frac{\partial \Gamma}{\partial \gamma}\right|_{\bar{i}=\frac{i_{\ell}+i_{d}}{2}} & =\theta\left[1-F\left(\hat{R}^{+}\right)+F\left(\hat{R}^{-}\right)\right]>0 \\
\left.\frac{\partial \Gamma}{\partial\left(i_{\ell}-i_{d}\right)}\right|_{\bar{i}=i_{\ell}} & =-\beta \theta \frac{\gamma}{2 \beta} f\left(R^{+}\right) \frac{1}{\beta\left(i_{\ell}-i_{d}\right)}+\theta\left[1-F\left(\frac{\gamma}{\beta\left(i_{\ell}-i_{d}\right)}\right)\right] \\
\left.\frac{\partial \Gamma}{\partial\left(i_{\ell}-i_{d}\right)}\right|_{\bar{i}=i_{d}} & =-\beta \theta \frac{\gamma}{2 \beta} f\left(R^{-}\right) \frac{1}{\beta\left(i_{\ell}-i_{d}\right)}+\theta F\left(\frac{-\gamma}{\beta\left(i_{\ell}-i_{d}\right)}\right)
\end{aligned}
$$

and $\Gamma$ increases with $\gamma$ if and only if

$$
2>\frac{\gamma}{\beta\left(i_{\ell}-i_{d}\right)} \frac{f\left(\frac{\gamma}{\beta\left(i_{\ell}-i_{d}\right)}\right)}{\left[1-F\left(\frac{\gamma}{\beta\left(i_{\ell}-i_{d}\right)}\right)\right]} .
$$

Finally $\bar{N}_{S}$ is implicitly given by

$$
\frac{\sigma \beta(1-\sigma)}{\left[(1-\sigma) \bar{N}_{S}+\sigma\right]} \frac{\Gamma}{\left[1-\beta(1-\sigma)\left(1-\bar{N}_{S}(1-\theta)\right)\right]}=\kappa .
$$

The LHS is decreasing in $N_{S}$ and increasing in $\Gamma$ and $\theta$. Hence $\bar{N}_{S}$ is increasing in $i_{\ell}-i_{d}, \theta$, and the variance of the shock $\xi$, and it is decreasing in $\kappa$. 


\section{E Exiting from the floor}

In section (5.3), we derive the transition path when the central bank announces at time $T$ it will exit from the floor at $T+1$, and when single $S$ banks strictly prefer to search. Here we consider the case when single $S$ banks are indifferent between searching and not for all $t \geq \tilde{T}$ (for some $\tilde{T}$ ). To solve for the transition path, notice that

$$
\begin{aligned}
v_{1}(1, t)-v_{1}(0, t)= & v_{2}(0,1, \mathbf{0}, t)-v_{2}(0,0, \mathbf{0}, t) \\
= & \Gamma+\beta E_{c}\left[c(1-\theta) \mathcal{V}_{t+1}\right]+\beta \bar{v}_{1}(1, t+1)-v(0, t) \\
= & \Gamma-\beta E_{c}\left[c(1-\theta)(1-\sigma)\left[v_{1}(1, t+1)-v_{1}(0, t+1)\right]\right]+\beta \bar{v}_{1}(1, t+1) \\
& -\max \left\{\beta v_{1}(0, t+1),-\kappa+N_{L}(t) \beta \bar{v}_{1}(1, t+1)+\left(1-N_{L}(t)\right) \beta v_{1}(0, t+1)\right\}
\end{aligned}
$$

(again, single banks search at $t$ but only get the benefit at $t+1$, so what matters is the number of available $L$ banks at date $t)$ and using $\bar{v}_{1}(1, t)=(1-\sigma) v_{1}(1, t)+\sigma v_{1}(0, t)$ and indifference to search,

$$
\begin{aligned}
& v_{1}(1, t)-v_{1}(0, t) \\
= & \Gamma+\beta(1-\sigma)\left[v_{1}(1, t+1)-v_{1}(0, t+1)\right]\left\{1-E_{c}[c(1-\theta)]\right\}
\end{aligned}
$$

The indifference condition gives us

$$
\beta v_{1}(0, t+1)=-\kappa+N_{L}(t) \beta \bar{v}_{1}(1, t+1)+\left(1-N_{L}(t)\right) \beta v_{1}(0, t+1)
$$

and using the expression for $\bar{v}_{1}(1, t+1)$, we get

$$
v_{1}(1, t+1)-v_{1}(0, t+1)=\frac{\kappa}{\beta(1-\sigma) N_{L}(t)} .
$$

Replacing this expression in (E.1), as well as $E_{c}[c]=N_{S}(t)$, we obtain

$$
v_{1}(1, t)-v_{1}(0, t)=\Gamma+\frac{\kappa}{N_{L}(t)}\left\{1-N_{S}(t)(1-\theta)\right\} .
$$


Since we assumed that single banks are always indifferent to searching after date $\tilde{T}$, we also have

$$
v_{1}(1, t)-v_{1}(0, t)=\frac{\kappa}{\beta(1-\sigma) N_{L}(t-1)} .
$$

Combining the last two equations and rearranging terms, we obtain a first difference equation

$$
N_{S}(t)=\frac{1}{\kappa(1-\theta)}\left[\Gamma N_{L}(t)+\kappa-\frac{\kappa N_{L}(t)}{\beta(1-\sigma) N_{L}(t-1)}\right],
$$

and the law of motion for $N_{L}(t+1)$ gives us a second,

$$
N_{L}(t+1)=1-\left[\left(1-N_{L}(t)\right)(1-\sigma)+N_{L}(t) N_{S}(t)(1-\sigma)\right] .
$$

These two difference equations define a second-order difference equation for $N_{L}(t)$ :

$N_{L}(t+1)=1-(1-\sigma)\left[1-N_{L}(t)+\frac{N_{L}(t)}{\kappa(1-\theta)}\left[\Gamma N_{L}(t)+\kappa-\frac{\kappa N_{L}(t)}{\beta(1-\sigma) N_{L}(t-1)}\right]\right]$

from which we obtain the transition path to the new steady state. 